\title{
Numerical Solution of the Ericksen-Leslie Model for Liquid Crystalline Polymers Free Surface Flows
}

\author{
Pedro A. Cruz \\ Sciences and Biotechnology \\ Federal University of Tocantins, Gurupi - To - Brasil \\ e-mail:pedrocruz@uft.edu.br \\ Murilo F. Tomé ${ }^{* \star}$ \\ Department of Applied Mathematics and Statistics \\ University of São Paulo, 13560-970, São Carlos - SP - Brazil \\ e-mail:murilo@icmc.usp.br \\ Sean McKee \\ Department of Mathematics and Statistics, University of Strathclyde, \\ Glasgow, Scotland \\ e-mail: sean.mckee@maths.strath.ac.uk \\ Iain Stewart \\ Faculty of Science, University of Strathclyde, Scotland \\ i.w.stewart@strath.ac.uk
}

\begin{abstract}
In this paper we present a finite difference method on a staggered grid for solving two-dimensional free surface flows of liquid crystalline polymers governed by the Ericksen-Leslie dynamic equations. The numerical technique is based on a projection method and employs Cartesian coordinates. The technique solves the governing equations using primitive variables for velocity, pressure, extra-stress tensor and the director. These equations are nonlinear partial differential equations consisting of the mass conservation equation and the balance laws of linear and angular momentum. Code verification and convergence estimates are effected by solving a flow problem on 5 different meshes. Two free surface problems are tackled: a jet impinging on a flat surface and injection molding. In the first case the buckling phenomenon is examined and shown to be highly dependent on the elasticity of the fluid. In the second case, injection molding of two differently shaped containers is carried out and the director is shown to be strongly dependent on its orientation at the boundaries.
\end{abstract}

ઋ Corresponding author 
Key words: Ericksen-Leslie model, Liquid crystalline polymers, Free-surface flows, Injection molding process, Finite difference

\section{Introduction}

Nematic liquid crystals and nematic liquid crystalline polymers (LCPs) are anisotropic materials; they are important engineering materials because of the wide range of their applications $([5,51])$. For example, nematic LCPs have been spun into high strength fibers which were used as airbags that cushioned the landing of NASAs highly successful missions to Mars ([17]). When nematic liquid crystalline polymers are used for industrial products, the molecular orientation within the products is important because their characteristics are dependent on their arrangement. Polymer processing principally involves injection molding. The direction or alignment of the molecules is dependent on the flow process and, after solidification, this alignment is maintained. Since the material and the mechanical strength of the finished product are highly dependent on their molecular alignment, it is vital to have a good understanding of the history of both the flow pattern and the molecular orientations.

Ericksen's $[18,19,20,21]$ transversely isotropic fluid, which was the first continuum theory for liquids with an intrinsic orientation, has been the basis for all subsequent developments of the continuum theory. The common feature of all such theories is that the stress depends on an orientational field in addition to the velocity gradients; the orientation is described by the distribution of a unit vector known as the director in some theories, and by the distribution of an orientation tensor in others. By making use of Ericksen's ideas, Leslie $[38,39]$ managed to formulate the constitutive equations and therefore complete the dynamic theory for nematic liquid crystals. This led to the celebrated Ericksen-Leslie dynamic theory of nematic liquid crystals about which this paper is concerned.

At present, Ericksen-Leslie theory [56] and Doi's theory [16,32] have become the two most popular constitutive theories describing the behavior of liquid crystals. Nematic liquid crystals have both elastic and viscous properties, and their molecular orientation is affected by the particular flow field. Many studies employing the Ericksen-Leslie equations have been undertaken (e.g. [9,11]). Others like Forest et al. [23,24] have employed Ericksen-Leslie equations to analise the flow induced behavior of nematic LCPs. Doi and Edwards [16] derived their model from microscopic molecular theory: it is a kinetic model for rod-like polymers. In the limit of low shear rates they demonstrated that it could be reduced to the Ericksen-Leslie theory. However, the Doi and Edwards model is complicated and not particularly suited for large scale simulations of 
complex problems.

There have been a number of experimental and theoretical studies of flow of low molar mass liquid crystals in complex geometries (e.g. $[2,31,33,47])$. With regard to the flow of liquid crystalline polymers, Kawaguchi and Denn [34] performed visualization of the flow of a thermotropic fluid in a tube with conical contraction while Cinader and Burghardt [13] used wide-angle X-ray crystalography to study LCP orientation in plane contraction and expansion. Many other authors have employed the Ericksen-Leslie equations to compute the flow behavior of LCPs (and nematic liquid crystals) (e.g. $[3,7,9,10]$ ). Cruz et. al. ([15]) developed a methodology for nematic liquid crystal flows in a confined region. This work extends Cruz et. al. ([15]) method to nematic LCPs with free surface flows; the extension, however, is non-trivial.

This paper, therefore is concerned with the development of a finite difference algorithm capable of efficiently solving complex free surface flows using the Ericksen-Leslie model. It is organised as follows: Section 2 presents the governing equations; Section 3 discusses the boundary conditions; while Section 4 describes the details of the numerical algorithm. In Section 5 a jet hitting a flat rigid plate is simulated on five different meshes to provide an indication that the code is working correctly and converging. Section 6 studies the jet buckling phenomenon and in particular addresses the role of elasticity in LCPs while Section 7 treats the problems of mold filling and shows how the director orientation is affected by the boundary conditions. Concluding remarks are then provided in Section 8. 


\section{Governing equations}

To facilitate the presentation of the equations, we shall employ the Einstein summation convention by which a comma between indices indicates partial differentiation with respect the variable it precedes. For example, $u_{, x}, n_{j, y}$ denote $\partial u / \partial x, \partial n_{j} / \partial y$ and so on.

The governing equations for two-dimensional Cartesian incompressible flows of nematic liquid crystalline polymers are the nondimensional mass conservation and momentum equations which can be written as (for details, see Cruz et al. [15], equations (41)-(49) with the term involving $H$ omitted).

$$
\begin{aligned}
u_{, x}+v_{, y} & =0 \\
u_{t}+u u_{, x}+v u_{, y} & =-p_{, x}-w_{F, x}+R_{j} n_{j, x} \\
& +\frac{1}{R e}\left[u_{, x x}+u_{, y y}\right]+\frac{1}{R e}\left[\Phi_{, x}^{x x}+\Phi_{, y}^{x y}\right]+\frac{1}{F r^{2}} g_{x}, \\
v_{t}+u v_{, x}+v v_{, y} & =-p_{, y}-w_{F, y}+R_{j} n_{j, y} \\
& +\frac{1}{R e}\left[v_{, x x}+v_{, y y}\right]+\frac{1}{R e}\left[\Phi_{, x}^{y x}+\Phi_{, y}^{y y}\right]+\frac{1}{F r^{2}} g_{y},
\end{aligned}
$$

where the terms $R_{j} n_{j, x}$ and $R_{j} n_{j, y}$, are given by

$$
\begin{aligned}
R_{j} n_{j, x} & =\frac{1}{R e}\left\{-\gamma_{1} \phi_{, x}\left[\phi_{, t}+u \phi_{, x}+v \phi_{, y}+\frac{1}{2}\left(u_{, y}-v_{, x}\right)\right]\right. \\
& \left.-\frac{1}{2}\left[\gamma_{2} \phi_{, x} \cos (2 \phi)\left(u_{, y}+v_{, x}\right)+\gamma_{2} \phi_{, x} \sin (2 \phi)\left(u_{, x}-v_{, y}\right)\right]\right\}, \\
R_{j} n_{j, y} & =\frac{1}{R e}\left\{-\gamma_{1} \phi_{, y}\left[\phi_{, t}+u \phi_{, x}+v \phi_{, y}+\frac{1}{2}\left(u_{, y}-v_{, x}\right)\right]\right. \\
& \left.-\frac{1}{2}\left[\gamma_{2} \phi_{, y} \cos (2 \phi)\left(u_{, y}+v_{, x}\right)+\gamma_{2} \phi_{, y} \sin (2 \phi)\left(u_{, x}-v_{, y}\right)\right]\right\},
\end{aligned}
$$

Equations (1a)-(1c) must be complemented with the elastic energy and the angular momentum equations given by (see [15])

$$
\begin{gathered}
w_{F}=\frac{1}{2} \frac{1}{\operatorname{Re}} \frac{1}{\operatorname{Er}}\left[\left(\phi_{, x}\right)^{2}+\left(\phi_{, y}\right)^{2}\right] \\
\left\{\begin{array}{l}
\phi_{t}=F(u, v, \phi) \\
F(u, v, \phi)=-u \phi_{, x}-v \phi_{, y}+\frac{1}{\operatorname{Er} \gamma_{1}}\left[\phi_{, x x}+\phi_{, y y}\right] \\
-\frac{1}{2} \frac{\gamma_{2}}{\gamma_{1}}\left[\left(u_{, y}+v_{, x}\right) \cos (2 \phi)+\left(v_{, y}-u_{, x}\right) \sin (2 \phi)\right] \\
-\frac{1}{2}\left(u_{, y}-v_{, x}\right) .
\end{array}\right.
\end{gathered}
$$


The constants appearing in equations (1a)-(4) are: $\phi_{0}$ is a given constant angle, $\gamma_{1}$ is the twist or rotational viscosity and $\gamma_{2}$ is the torsion coefficient of viscosity.

In equations (1a)-(4), the nondimensional numbers are the Reynolds number $\left(R e=\frac{\rho U L}{\eta}\right)$, the Ericksen number $\left(E r=U L \frac{\eta}{K}\right)$ and the Froude number $\left(F r=\frac{U}{\sqrt{g L}}\right)$. The appropriate scalings $U, L, \rho$ and $\eta$ are associated with velocity, length, density and viscosity, respectively, while $K$ is an elastic constant and $g$ is the gravitational constant. Complete details about the derivation of these equations are provided in Cruz et al. [15] and for this reason they are not presented here.

In equations above, $u_{i}=(u(x, y, t), v(x, y, t))$ is the velocity field, $p=p(x, y, t)$ is the pressure, $w_{F}(x, y, t)$ is the elastic energy density and $\phi=\phi(x, y, t)$ is the orientation angle of the director $n_{i}=(\cos (\phi), \sin (\phi))$.

The momentum equations (1b) and (1c) were obtained after the viscous stress tensor was split into the sum of a Newtonian and a non-Newtonian stress tensor, $\boldsymbol{\Phi}_{m l}$, as follows:

$$
\begin{aligned}
& S^{x x}=\frac{1}{R e}\left[2 u_{, x}+\Phi^{x x}\right], \\
& \boldsymbol{\Phi}_{\boldsymbol{m l}}=\left[\begin{array}{cc}
\Phi^{x x} & \Phi^{x y} \\
\Phi^{y x} & \Phi^{y y}
\end{array}\right], \quad \begin{aligned}
S x y & =\frac{1}{R e}\left[\left(u_{, y}+v_{, x}\right)+\Phi^{x y}\right], \\
S^{y x} & =\frac{1}{R e}\left[\left(u_{, y}+v_{, x}\right)+\Phi^{y x}\right],
\end{aligned} \\
& S^{y y}=\frac{1}{R e}\left[2 v_{, y}+\Phi^{y y}\right] \text {, }
\end{aligned}
$$

where $\Phi^{x x}, \cdots, \Phi^{y y}$ are the components of the non-Newtonian stress tensor $\boldsymbol{\Phi}_{m l}$ given by (for details, see [15]) 


$$
\begin{aligned}
\Phi^{x x}= & \alpha_{1} \cos ^{2} \phi\left[u_{, x} \cos ^{2} \phi+v_{, y} \sin ^{2} \phi+\frac{1}{2}\left(u_{, y}+v_{, x}\right) \sin (2 \phi)\right] \\
& -\left(\alpha_{2}+\alpha_{3}\right) \sin \phi \cos \phi\left[\phi_{t}+u \phi_{, x}+v \phi_{, y}+\frac{1}{2}\left(u_{, y}-v_{, x}\right)\right] \\
& +\left(\alpha_{5}+\alpha_{6}\right)\left[u_{, x} \cos ^{2} \phi+\frac{1}{2} \sin \phi \cos \phi\left(u_{, y}+v_{, x}\right)\right] \\
\Phi^{x y}= & \alpha_{1} \sin \phi \cos \phi\left[u_{, x} \cos ^{2} \phi+v_{, y} \sin ^{2} \phi+\frac{1}{2}\left(u_{, y}+v_{, x}\right) \sin (2 \phi)\right] \\
& +\left(\alpha_{3} \cos ^{2} \phi-\alpha_{2} \sin ^{2} \phi\right)\left[\phi_{t}+u \phi_{, x}+v \phi_{, y}+\frac{1}{2}\left(u_{, y}-v_{, x}\right)\right] \\
& +\frac{1}{2}\left(\alpha_{5} \sin ^{2} \phi+\alpha_{6} \cos ^{2} \phi\right)\left(u_{, y}+v_{, x}\right) \\
& +\left(\alpha_{5} u_{, x}+\alpha_{6} v_{, y}\right) \sin \phi \cos \phi \\
& \alpha_{1} \sin ^{y x} \cos \phi\left[u_{, x} \cos ^{2} \phi+v_{, y} \sin ^{2} \phi+\frac{1}{2}\left(u_{, y}+v_{, x}\right) \sin (2 \phi)\right] \\
& +\left(\alpha_{2} \cos ^{2} \phi-\alpha_{3} \sin ^{2} \phi\right)\left[\phi_{t}+u \phi_{, x}+v \phi_{, y}+\frac{1}{2}\left(u_{, y}-v_{, x}\right)\right] \\
& +\frac{1}{2}\left(\alpha_{5} \cos ^{2} \phi+\alpha_{6} \sin ^{2} \phi\right)\left(u_{, y}+v_{, x}\right) \\
& +\left(\alpha_{5} v_{, y}+\alpha_{6} u_{, x}\right) \sin \phi \cos \phi, \\
& \\
\Phi^{y y}= & \alpha_{1} \sin ^{2} \phi\left[u_{, x} \cos ^{2} \phi+v_{, y} \sin ^{2} \phi+\frac{1}{2}\left(u_{, y}+v_{, x}\right) \sin (2 \phi)\right] \\
& +\left(\alpha_{2}+\alpha_{3}\right) \sin \phi \cos \phi\left[\phi_{t}+u \phi_{, x}+v \phi_{, y}+\frac{1}{2}\left(u_{, y}-v_{, x}\right)\right] \\
& +\left(\alpha_{5}+\alpha_{6}\right)\left[v_{, y} \sin ^{2} \phi+\frac{1}{2} \sin \phi \cos \phi\left(u_{, y}+v_{, x}\right)\right] .
\end{aligned}
$$

In the equations (9a)-(9d) above, the Leslie viscosities $\alpha_{1}, \ldots, \alpha_{6}$ have been scaled by the factor $\eta$.

In order to simulate the flow of a nematic liquid crystal or nematic LCPs one needs to be able to solve the incompressibility condition (1a) together with equations (1b), (1c) and (4) subject to appropriate initial and boundary conditions. 


\section{Boundary conditions}

Four types of boundaries are considered: rigid boundaries, prescribed inflows, outflows, and free surfaces. The boundary conditions are specified as follows. For two-dimensional flows, let $\boldsymbol{\nu}=\left(\nu_{x}, \nu_{y}\right)^{T}$ and $\boldsymbol{\mu}=\left(-\nu_{y}, \nu_{x}\right)^{T}$ denote unit normal and tangential vectors to the boundary, respectively. Then, the velocity $\mathbf{u}=(u, v)^{T}$, on the boundary, is specified according to:

- rigid boundaries : $\mathbf{u}^{T} \cdot \boldsymbol{\nu}=0$ and $\mathbf{u}^{T} \cdot \boldsymbol{\mu}=0$. This implies that $u=v=0$ on the boundary.

- prescribed inflows: $\mathbf{u}^{T} \cdot \boldsymbol{\nu}=U_{\mathrm{inf}}$ and $\mathbf{u}^{T} \cdot \boldsymbol{\mu}=0$ which gives the $(2 \times 2)$ linear system (since $\mu_{x}=-\nu_{y}$ and $\mu_{y}=\nu_{x}$ )

$$
\left\{\begin{array} { l } 
{ u \nu _ { x } + v \nu _ { y } = U _ { \mathrm { inf } } , } \\
{ - u \nu _ { y } + v \nu _ { x } = 0 . }
\end{array} \quad \left\{\begin{array}{l}
\text { which yields } \\
u=U_{\mathrm{inf}} \nu_{x} \\
v=U_{\mathrm{inf}} \nu_{y}
\end{array}\right.\right.
$$

- outflows: Neumann conditions are imposed, $(\nabla u) \cdot \boldsymbol{\nu}=0$ and $(\nabla v) \cdot \boldsymbol{\nu}=0$, specifically, $\frac{\partial u}{\partial x} \nu_{x}+\frac{\partial u}{\partial y} \nu_{y}=0$ and $\frac{\partial v}{\partial x} \nu_{x}+\frac{\partial v}{\partial y} \nu_{y}=0$.

- free surfaces: On free surfaces we neglect surface tension forces so that the correct boundary conditions to be satisfied are (see Batchelor [4]):

$$
\begin{aligned}
& \boldsymbol{\nu}^{T} \cdot \mathbf{t} \cdot \boldsymbol{\nu}=0 \\
& \boldsymbol{\mu}^{T} \cdot \mathbf{t} \cdot \boldsymbol{\nu}=0
\end{aligned}
$$

where the total stress tensor $\boldsymbol{t}_{\boldsymbol{m} \boldsymbol{l}}$ is given by

$$
\boldsymbol{t}_{\boldsymbol{m} \boldsymbol{l}}=-p \boldsymbol{\delta}_{\boldsymbol{m} \boldsymbol{l}}+\frac{1}{R e}\left[u_{m, l}+u_{l, m}\right]+\frac{1}{R e} \boldsymbol{\Phi}_{\boldsymbol{m} \boldsymbol{l}} .
$$

Thus, for two-dimensional Cartesian flows, conditions (10a) and (10b) can be rewritten as

$$
\begin{gathered}
\tilde{p}=\frac{2}{R e}\left[u_{, x} \nu_{x}^{2}+v_{, y} \nu_{y}^{2}+\nu_{x} \nu_{y}\left(u_{, y}+v_{, x}\right)\right] \\
\quad+\frac{1}{R e}\left[\Phi^{x x} \nu_{x}^{2}+\Phi^{y y} \nu_{y}^{2}+\nu_{x} \nu_{y}\left(\Phi^{x y}+\Phi^{y x}\right)\right] \\
0=\frac{1}{R e}\left[2\left(u_{, x}-v_{, y}\right) \nu_{x} \nu_{y}+\left(u_{, y}+v_{, x}\right)\left(\nu_{y}^{2}-\nu_{x}^{2}\right)\right] \\
\quad+\frac{1}{R e}\left[\Phi^{x y} \nu_{y}^{2}-\Phi^{y x} \nu_{x}^{2}+\nu_{x} \nu_{y}\left(\Phi^{x x}-\Phi^{y y}\right)\right] .
\end{gathered}
$$


Figure 1 illustrates the types of boundaries within a computational domain.

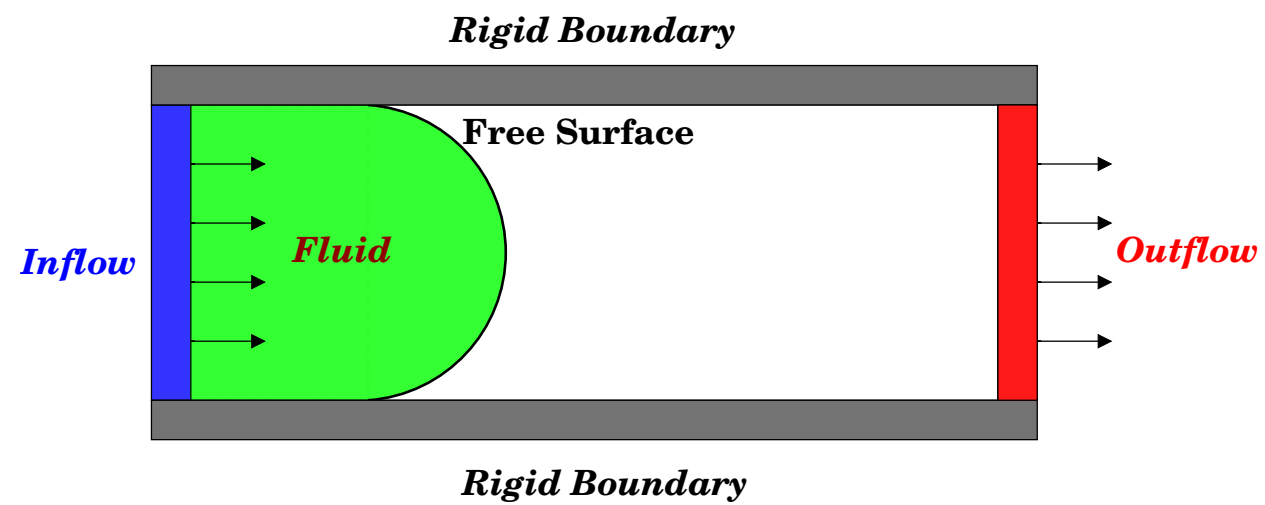

Figure 1. Types of boundaries considered.

\section{GENSMAC-ERL-Model2D - ERicksen-Leslie}

A typical cell is displayed in Fig. 2a and a staggered grid is employed whereby $p_{i j}, \phi_{i j}$ and $\Phi_{i j}$ are placed at the centre of the cell while the velocities are displaced by half a grid length and sit on the cell faces. For flows containing free surfaces, a technique that defines the fluid contour is required. The technique employed in this work is a modified Marker-and-Cell method that was initially introduced by Harlow and Welch [30] and was further developed by Tomé et al. [57,60] who have simulated free surface flows of Newtonian [59] and non-Newtonian [63] fluids. The markers or massless particles are only retained in the surface cells: this necessitates removal and addition of particles while maintaining mass conservation (for details see [41]). In this technique, the contour of the fluid (and, in particular, the free surface) is defined by a set of massless particles that moves with the local fluid velocity. The free surface of the fluid is visualized by connecting these particles by straight lines and the corresponding volume of fluid is that encapsulated area defined by the closed surface (see Fig. 2a). When surface tension is present a more sophisticated approach is required (see [41]). To implement this methodology it was necessary to categorise the cells within the domain into several groups as follows: (see Fig. 3):

- Rigid boundary (B): cells that define the location of rigid contours;

- Inflow boundary (I): cells that model 'fluid entrances' ('inflows');

- Outflow boundary (O): cells that define 'fluid exits' ('outflows');

- Empty cells (E): cells that do not contain fluid;

- Full cells (F): cells that contain fluid but have no contact with E-cell faces;

- Surface cells (S): cells that contain fluid and have at least one face in contact with E-cell faces. 
The solution of equations (1a), (1b)-(1c), (3) and (4) is obtained by splitting the calculational cycle into two sequential phases: in the first phase, the velocity field $u_{m}\left(x_{k}, t_{n}\right)$ and the orientation angle of the director $\phi\left(x_{k}, t_{n}\right)$ are used to calculate the new velocity field $u_{m}\left(x_{k}, t_{n+1}\right)$ and the new pressure $p\left(x_{k}, t_{n+1}\right)$ while, in the second phase, the updated velocity field is employed to determine the orientation angle of the director $\phi\left(x_{k}, t_{n+1}\right)$ and the non-Newtonian stress tensor $\boldsymbol{\Phi}_{\boldsymbol{m l}}\left(x_{k}, t_{n+1}\right)$.

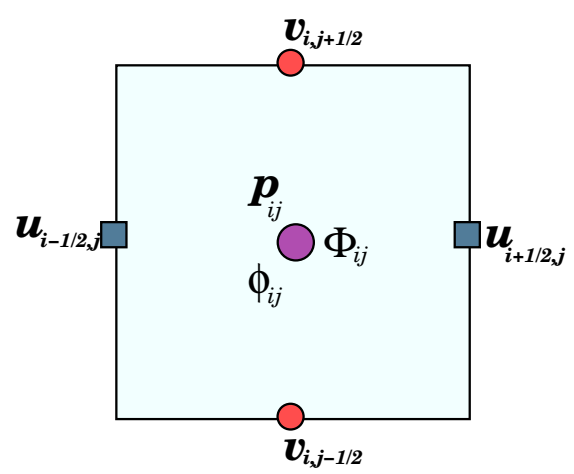

(a)

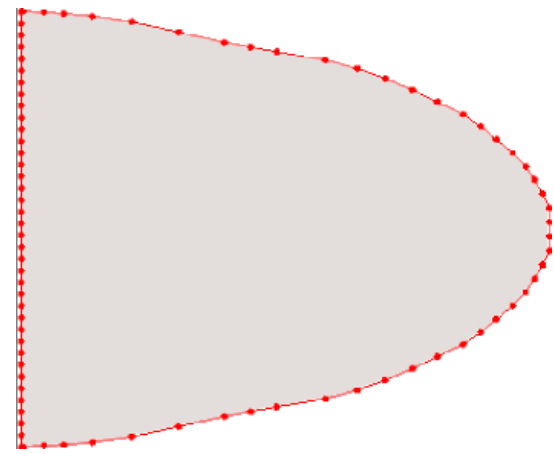

(b)

Figure 2. (a) Representation of fluid free surface (line conecting the particles) and volume of fluid (grey area), (b) Description of the staggered cell.

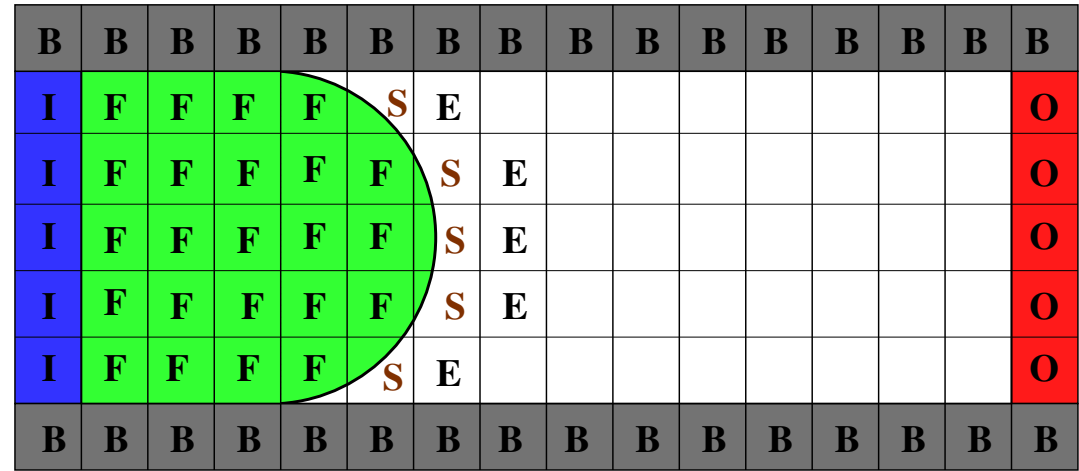

Figure 3. Type of cells considered within the computational domain.

\subsection{Calculation of $u_{m}\left(x_{k}, t_{n+1}\right)$ and $p\left(x_{k}, t_{n+1}\right)$}

Equation (1a) together with equations (1b)-(1c) are solved by an implicit method used by Oishi et al. [43] to simulate flows of XPP fluids. This technique solves two linear systems to obtain a tentative velocity field $\widetilde{u}_{m}$, followed by the solution of a Poisson equation to ensure incompressibility throughout. One feature of the solution of the Poisson equation is that it is coupled with the pressure condition on the free surface (12a) through the incompressibility condition (1a) allowing the calculation of the pressure implicitly. This results 
in a nonsymmetric system of linear equations which are solved by the biconjugate gradient method. Details of the algorithm employed are given next.

Let $u_{m}\left(x_{k}, t_{n}\right)=u_{m}{ }^{(n)}, p\left(x_{k}, t_{n}\right)=p^{(n)}, \boldsymbol{\Phi}_{m \boldsymbol{l}}\left(x_{k}, t_{n}\right)=\boldsymbol{\Phi}_{\boldsymbol{m l}}{ }^{(n)}$ and the markers' positions $x_{k}\left(t_{n}\right)=x_{k}{ }^{(n)}$ be known. Then $u_{m}\left(x_{k}, t_{n+1}\right)=u_{m}{ }^{(n+1)}$ and the pressure $p\left(x_{k}, t_{n+1}\right)=p^{(n+1)}$ at time $t_{n+1}$ are obtained as follows.

Step 1: Using the values of $u_{m}\left(x_{k}, t_{n}\right)$ and $\phi\left(x_{k}, t_{n}\right)$, solve Eq. (3) for $w_{F}\left(x_{k}, t_{n}\right)$ and calculate the derivatives $w_{F, m}\left(x_{k}, t_{n}\right)$ and compute $\boldsymbol{\Phi}_{m l}\left(x_{k}, t_{n}\right)$ from equations (9a)-(9d) and $R_{l} n_{l, m}\left(x_{k}, t_{n}\right)$ using equations (2a) and (2b), respectively.

Step 2: Calculate an intermediate velocity field $\widetilde{u}_{m}$ by

$$
\frac{\partial \widetilde{u}_{m}}{\partial t}=-\left(u_{l} u_{m}\right)_{, l}-p_{, m}^{(n)}-w_{F, m}+R_{l} n_{l, m}+\frac{1}{R e}\left[\left(u_{m, l}\right)_{l}+\Phi_{m l, l}\right]+\frac{1}{F r^{2}} g_{x},
$$

The boundary conditions for $\widetilde{u}_{m}^{(n+1)}$ are those imposed on $u_{m}^{(n+1)}$.

This equation is applied to each $\mathbf{F}$ and $\mathbf{S}$-cells within the domain leading to two sparse symmetric positive definite linear systems that are solved by the conjugate gradient method, using the velocity value at the previous time step as an initial guess which, in practice, if $\delta t$ is small enough, precludes the need for preconditioning. It can be shown that $\widetilde{u}_{m}^{(n+1)}$ contains the correct vorticity at time $t_{n+1}[58]$ but it does not ensure incompressibility. For that reason, a potential function $\psi^{(n+1)}$ is employed such that

$$
\begin{aligned}
& \left(\psi_{m, l}\right)_{l}^{(n+1)}=\left(\widetilde{u}_{m}\right)_{, m}^{(n+1)} . \\
& u_{m}{ }^{(n+1)}=\widetilde{u}_{m}^{(n+1)}-\psi_{, m}^{(n+1)},
\end{aligned}
$$

Thus, after solving the Poisson equation (14a) the velocity is obtained from equation (14b). This Poisson equation is applied to every F-cell subject to a homogeneous Neumann condition on inflows and rigid boundaries and a homogeneous Dirichlet condition $(\psi=0)$ on outflows and free surfaces. The pressure is given by (see Tomé et al. [58])

$$
p^{(n+1)}=p^{(n)}+\frac{\psi^{(n+1)}}{\delta t} .
$$

Step 3: Implicit calculation of the pressure on the free surface When solving the Poisson equation (14a), the Dirichlet boundary condition for $\psi$ on the free surface determines that it is zero on surface cells $\mathbf{S}$. In this work we employ the implicit technique of Oishi et al. [43] in which additional equations for $\psi^{(n+1)}$ on surface cells $\mathbf{S}$ are obtained. This is achieved by writing 
equation $(12 \mathrm{a})$ in the form

$$
\begin{aligned}
p^{(n+1)}= & \frac{2}{R e}\left[\nu_{x}^{2} u_{, x}^{(n+1)}+\nu_{y}^{2} v_{, y}^{(n+1)}+\nu_{x} \nu_{y}\left(u_{, y}^{(n+1)}+v_{, x}^{(n+1)}\right)\right] \\
& +\frac{1}{\operatorname{Re}}\left[\nu_{x}^{2} \Phi^{x x}+\nu_{y}^{2} \Phi^{y y}+\nu_{x} \nu_{y}\left(\Phi^{x y}+\Phi^{y x}\right)\right] .
\end{aligned}
$$

and making use of the mass conservation equation (1a), together with equations (14b) and (15); then equation (16) may be rewritten as

$$
\begin{aligned}
& \frac{\psi^{(n+1)}}{\delta t}-\frac{2}{R e}\left[\psi_{, y y}^{(n+1)} \nu_{x}^{2}+\psi_{, x x}^{(n+1)} \nu_{y}^{2}-2 \psi_{, x y}^{(n+1)} \nu_{x} \nu_{y}\right] \\
& =\frac{2}{R e}\left[-\widetilde{v}_{, y}^{(n+1)} \nu_{x}^{2}-\widetilde{u}_{, x}^{(n+1)} \nu_{y}^{2}+\widetilde{u}_{, y}^{(n+1)}+\widetilde{v}_{, x}^{(n+1)} \nu_{x} \nu_{y}\right] \\
& +\frac{1}{R e}\left[\Phi^{x x} \nu_{x}^{2}+\nu_{x} \nu_{y}\left(\Phi^{x y}+\Phi^{y x}\right)+\Phi^{y y} \nu_{y}^{2}\right]-p^{(n)} .
\end{aligned}
$$

This equation is applied to every $\mathbf{S}$-cell. It is assumed that the mesh is sufficiently fine so that the free surface will cut a S-cell either on two-opposite faces (see Fig. 4a) or will intercept the cell on two adjacent faces (see Fig. 4b). In both cases, an approximation for the normal vector is determined by examining the $\mathbf{S}$-cell neighbours. For instance, considering the cell $\mathbf{S}_{\mathbf{i}, \mathbf{j}}$ displayed in Fig. 4a, we assume that the free surface is horizontal and then define the normal vector as $\boldsymbol{\nu}=(0,1)$ so that equation (17) reduces to

$$
\frac{R e}{\delta t} \psi_{\mathbf{i}, \mathbf{j}}^{(n+1)}-2\left(\psi_{\mathbf{i}, \mathbf{j}}\right)_{, x x}^{(n+1)}=-2\left(\widetilde{v}_{\mathbf{i}, \mathbf{j}}\right)_{, x}^{(n+1)}+\Phi_{\mathbf{i}, \mathbf{j}}^{y y}-\operatorname{Re} p_{\mathbf{i}, \mathbf{j}}^{(n)},
$$

while if we consider the cell $\mathbf{S}_{\mathbf{i}, \mathbf{j}}$ shown in Fig. $4 \mathrm{~b}$, then a $45^{\circ}$-sloped surface is assumed and we take $\boldsymbol{\nu}=\left(\frac{\sqrt{2}}{2}, \frac{\sqrt{2}}{2}\right)$. In this case, equation (17) becomes

$$
\begin{aligned}
\frac{R e}{\delta t} \psi_{\mathbf{i}, \mathbf{j}}^{(n+1)}+2\left(\psi_{\mathbf{i}, \mathbf{j}}\right)_{, x y}^{(n+1)} & =\left(\left(\widetilde{u}_{\mathbf{i}, \mathbf{j}}\right)_{, y}^{(n+1)}+\left(\widetilde{v}_{\mathbf{i}, \mathbf{j}}\right)_{, x}^{(n+1)}\right) \\
& +\frac{1}{2}\left(\Phi^{x x}+\Phi^{x y}+\Phi^{y x}+\Phi^{y y}\right)_{\mathbf{i}, \mathbf{j}}-\operatorname{Re} p_{\mathbf{i}, \mathbf{j}}^{(n)} .
\end{aligned}
$$

The code contains a check: if any one cut by the free surface does not fall clearly into one or other of these categories then a flag indicates that the mesh should be further refined.

These approximations simplify equation (17) which is then approximated by local finite differences. For other types of surface approximations (vertical or variations of $45^{\circ}$-sloped surfaces), the normal vector is obtained in a similar manner. The equations for $\psi$ on each $\mathbf{F}$ and $\mathbf{S}$-cells are grouped into a nonsymmetric sparse linear system that is solved by the bi-conjugate gradient 
method. Details of the finite difference equations (and the preconditioning applied) are provided in the works of Oishi et al. [43] and Tomé et al. [65].

Once the final velocities have been calculated, the positions of the markers $\left(\mathbf{x}_{\mathbf{p}}=\left(x_{p}, y_{p}\right)\right)$ that describe the fluid surface are updated by solving the ordinary differential equations

$$
\frac{d x_{p}}{d t}=u_{p}^{(n+1)} \quad \text { and } \quad \frac{d y_{p}}{d t}=v_{p}^{(n+1)}
$$

by the second order improved Euler's method. The velocities $u_{p}$ and $v_{p}$ are found from bilinear interpolation involving the nearest values of $u_{p}$ and $v_{p}$. Connecting these particles using straight lines provides the new fluid surface. Details on the particles' movement are given in Tomé and McKee [57].

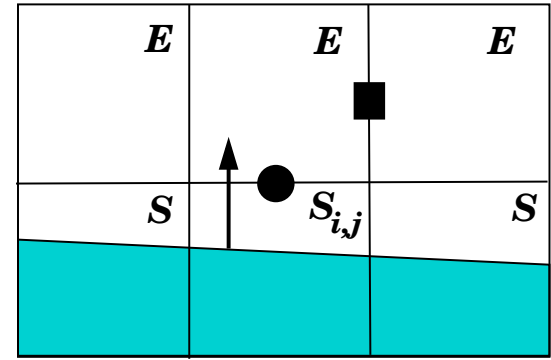

(a)

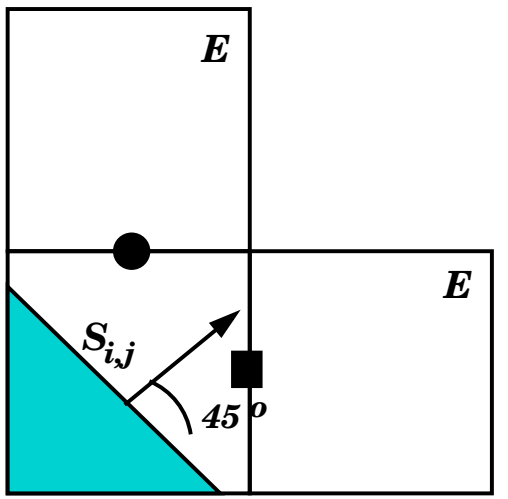

(b)

Figure 4. Free surface orientations. (a) horizontal surface: $\boldsymbol{\nu}=(0,1)^{T}$ and $\boldsymbol{\mu}=(-1,0)^{T}$; (b) $45^{\circ}$ sloped surface: $\boldsymbol{\nu}=\left(\frac{\sqrt{2}}{2}, \frac{\sqrt{2}}{2}\right)^{T}$ and $\boldsymbol{\mu}=\left(-\frac{\sqrt{2}}{2}, \frac{\sqrt{2}}{2}\right)^{T}$.

Step 4: Calculation of the time-step size: The use of an implicit method for the calculation of the velocities, while it is a distinct improvement over the explicit method previously employed, does not yield an unconditionally stable algorithm over all. There are essentially two restrictions on the choice of the time step: a viscous restriction that arises from a "Von Neumann" like condition and a Courant-Friedrichs-Lewy restriction; the latter really requiring that marker particles should only move, at most, from one cell to a neighbouring cell. 
We have found empirically that a good choice is

$$
\begin{aligned}
& \delta t=\boldsymbol{\Gamma} * \min \left\{\delta t_{M E S H}, \delta t_{C F L}\right\}, \\
& \text { where }\left\{\begin{array}{l}
\delta t_{\mathrm{MESH}}=\frac{h^{2}}{4}(\text { where, } h=\delta x=\delta y), \\
\delta t_{\mathrm{CFL}}=\min \left\{\frac{h}{\max |u|}, \frac{h}{\max |v|}\right\},
\end{array}\right.
\end{aligned}
$$

and $\boldsymbol{\Gamma}$ is a positive number such that $0<\boldsymbol{\Gamma}<1$.

\subsection{Computation of the angle $\phi$ and the non-Newtonian tensor $\mathbf{\Phi}$}

By using $u_{m}\left(x_{k}, t_{n+1}\right)$, the angle of the director $\phi\left(x_{k}, t_{n+1}\right)$ is obtained from equation (4); it is solved by the following second-order Runge-Kutta scheme:

$$
\left\{\begin{array}{l}
K_{1}=F\left(u^{(n+1)}, v^{(n+1)}, \phi^{(n)}\right) \\
\widehat{\phi}=\phi^{(n)}+\delta t K_{1} \\
K_{2}=F\left(u^{(n+1)}, v^{(n+1)}, \widehat{\phi}^{(n)}\right) \\
\phi^{(n+1)}=\phi^{(n)}+\frac{\delta t}{2}\left[K_{1}+K_{2}\right]
\end{array}\right.
$$

where the boundary conditions for $\phi$ will be discussed in the next section. The function $F$ is given in equation (4).

After calculating the angle of the director, the components of the non-Newtonian tensor $\boldsymbol{\Phi}$ are computed from equations (9a)-(9d).

\section{Simulation of a jet hitting a rigid plate}

In order to verify the code and demonstrate the convergence of the numerical method, a jet of diameter $D=0.4 \mathrm{~mm}$ containing the nematic LCP MBBA ${ }^{2}$ impacting a rigid surface was simulated and mesh refinement studies were performed. In these simulations, a computational domain of dimensions $W$ by $H(W=4 \mathrm{~mm}, H=6 \mathrm{~mm})$ was utilized (see Fig. 5$)$, and the five different meshes defined in Table 1 were employed. The properties of the nematic LCP fluid are displayed in Table 2. The remaining data used are displayed in Table 3 . The boundary conditions employed to calculate the director angle are those depicted in Fig. 5b.

$\overline{2}$ MBBA or N-(4-Methoxybensylidene)-4-butylaniline is an organic compound often used as a liquid crystal 
The results obtained in these simulations are presented in Fig. 6 which shows several snapshots taken at selected times during the simulation. By examining Fig. 6 we can observe that the surface profiles obtained on the coarse meshes approximate the surface profile of mesh $M_{5}$. Furthermore, the surface profiles of meshes $M_{3}, M_{4}$ and $M_{5}$ appear very close to each other. Therefore, these results give the impression at least that the numerical method for this particular free surface flow problem converges.

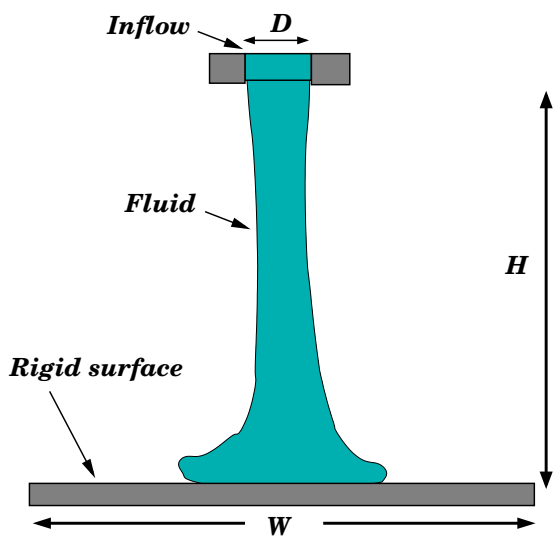

(a)

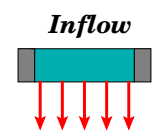

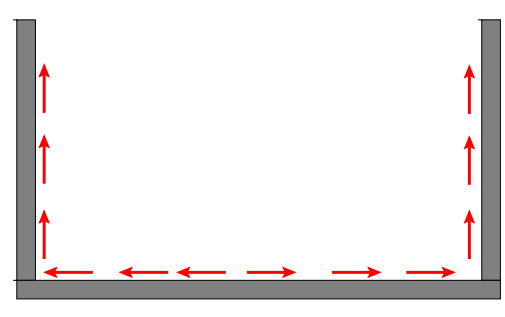

Rigid Surface

(b)

Figure 5. (a) Description of the domain used in the simulation, (b) Boundary conditions for the computation of the Director angle $\phi$.

Table 1

Meshes employed in the simulation of a jet hitting a rigid plate.

\begin{tabular}{|c|c|c|c|c|c|}
\hline Meshes & $\mathbf{M}_{1}:$ & $\mathbf{M}_{2}:$ & $\mathbf{M}_{3}:$ & $\mathbf{M}_{4}:$ & $\mathbf{M}_{5}:$ \\
$\delta x=\delta y$ & $\delta x=\frac{D}{8}$ & $\delta x=\frac{D}{16}$ & $\delta x=\frac{D}{20}$ & $\delta x=\frac{D}{32}$ & $\delta x=\frac{D}{40}$ \\
$D=0.4 \mathrm{~mm}$ & $80 \times 120$ & $160 \times 240$ & $200 \times 300$ & $320 \times 480$ & $400 \times 600$ \\
\hline
\end{tabular}

Table 2

Definition of the fluid used in the results of this paper. Physical parameters are in SI units.

\begin{tabular}{|ccccccc|}
\hline \multicolumn{8}{|c|}{$\rho=1088 \mathrm{~kg} \mathrm{~m}^{-3}$} \\
\hline Leslie viscosities & $\alpha_{1}$ & $\alpha_{2}$ & $\alpha_{3}$ & $\alpha_{4}$ & $\alpha_{5}$ & $\alpha_{6}$ \\
\hline MBBA near $25^{\circ} \mathrm{C}$ & -0.0181 & -0.1104 & -0.001104 & 0.0826 & 0.0779 & -0.0336 \\
\hline
\end{tabular}

Table 3

Data used in the simulation of a jet hitting a rigid plate.

\begin{tabular}{|ccccccccc|}
\hline$D$ & $U$ & $H$ & $W$ & $H / D$ & $R e$ & $K$ & $E r$ & $F r$ \\
\hline $0.4 \mathrm{~mm}$ & $0.04 \mathrm{~ms}^{-1}$ & $6 \mathrm{~mm}$ & $4 \mathrm{~mm}$ & 15 & 0.4 & $3.3 \times 10^{-8}$ & 20 & 0.64 \\
\hline
\end{tabular}



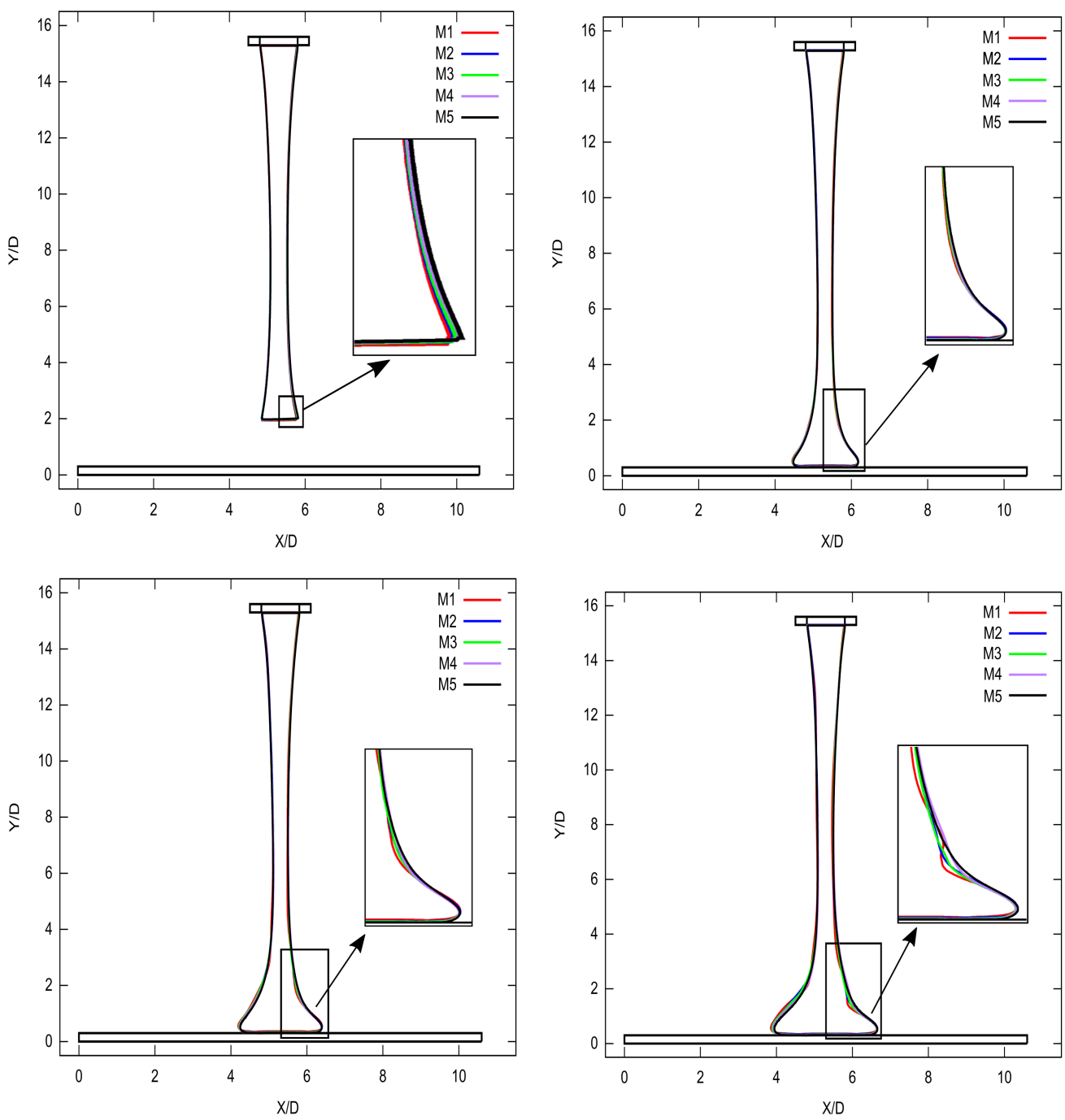

Figure 6. Free surface profiles obtained in the simulation of a jet flowing onto a rigid surface, using the nematic LCP fluid MBBA. Fluid flow visualizations at selected times on meshes $M_{1}, M_{2}, M_{3}, M_{4}$ and $M_{5}$. 


\section{Numerical simulation of jet buckling of nematic LCP fluids}

To demonstrate that the numerical method is capable of predicting free surface flow instabilities, we have explored numerically the phenomenon known as jet buckling. An example of this phenomenon is the coiling and folding of a thin thread of viscous fluid falling onto a rigid horizontal surface (see Fig. 7). It has been intensively studied by many researchers (e.g.[14,52,53]). This phenomenon is characterized by the formation of a physical instability that occurs after a viscous fluid jet impinges upon a rigid plate. This problem was experimentally and theoretically studied by Cruickshank and Munson [14] for Newtonian fluids, who discovered that this instability is influenced by the height $H$ between the nozzle and the rigid plate, jet width $D$ and Reynolds number Re. In their analyses, Cruickshank and Munson [14] found that a twodimensional Newtonian jet will undergo "jet buckling" if the conditions below are approximately satisfied

$$
R e<0.56 \text { and } H / D>10 \text {. }
$$

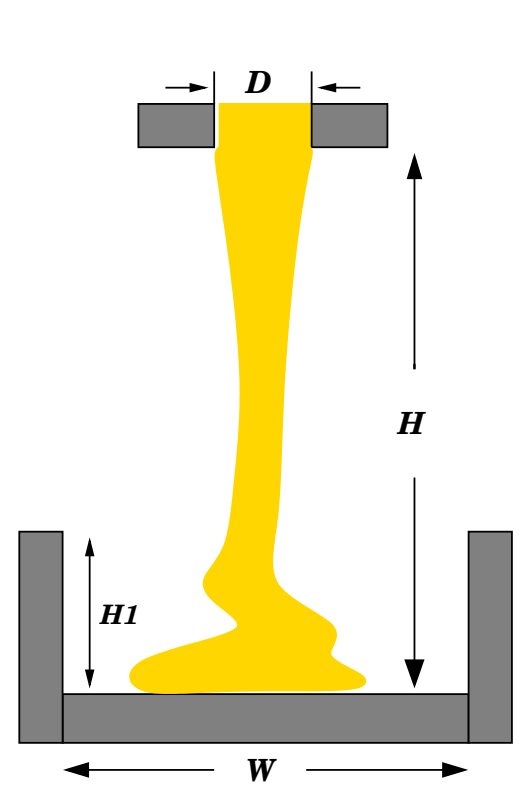

(a)

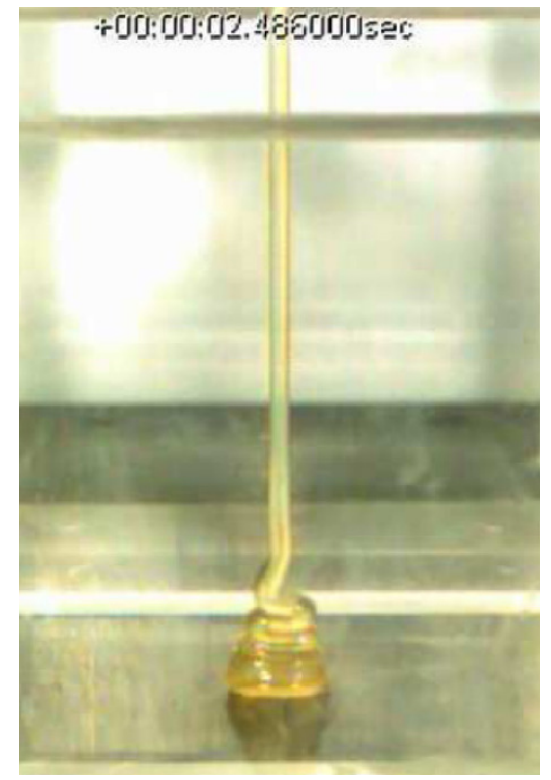

(b)

Figure 7. a) Description of the domain used in the simulation. b) Example of jet buckling of a Newtonian jet (from Tomé et al. [64]).

A number of researchers simulated this phenomenon in two and three dimensions employing Newtonian fluids and confirmed the conditions given by inequalities (23). Numerical simulations have been carried out by Tomé et al. [64] using a marker-and-cell finite difference method for Newtonian and Oldroyd-B fluids while Rafiee et al. [48], in the context of the SPH method, 
also presented numerical simulations of the jet buckling phenomenon for Newtonian and Oldroyd-B jets. More recently, Roberts and Rao [54], carried out an interesting computational study of continuous jets of shear thinning fluids impinging on a fluid surface.

We consider the simulation of the filling of a square container depicted in Fig. 7a. We are interested in whether Nematic Liquid Crystalline Polymers will undergo buckling during the filling process. The properties of the nematic LCP fluid employed in this simulation are described in Table 2 and the dimensions of the computational domain are displayed schematically in Fig. 7a. The mesh spacing was defined to be $\delta x=\delta y=D / 8=0.05 \mathrm{~mm}$ where $D=0.4 \mathrm{~mm}$ was the inlet diameter from which the jet issued. Gravity forces acted downwards with $g_{y}=-9.81 \mathrm{~ms}^{-2}$. The values of the nondimensional numbers, $R e, F r, E r$ and the velocity $U$ and the elastic constant $K$, involved in these simulations, are given in Table 4. Using the scaling velocities $U=0.0285$ and $U=0.057$ $\mathrm{ms}^{-1}$ leads to $R e=0.3$ and $R e=0.6$, respectively, and so the Cruickshank and Munson conditions (23) are satisfied only for $U=0.0285$. Thus, it is anticipated that a Newtonian jet will undergo buckling when $U=0.0285$ $\mathrm{ms}^{-1}$, but will not when $U=0.057$. For the jet containing the nematic LCP fluid, there would appear to be no asymptotic analysis of jet buckling.

The results of these simulations are presented in Figs. 8 and 9. We observe, from Fig. 8, that both Newtonian and nematic LCP jets buckle when $R e=0.3$. However, for $R e=0.6$, Fig. 9 shows that the Newtonian jet does not buckle while nematic LCP jet does: this is likely to be due to the elasticity of the fluid. To confirm this hypothesis, additional simulations were performed and are presented in the next section.

Table 4

Data employed in the simulation of an impinging jet of a nematic LCP fluid.

\begin{tabular}{|ccc|c|c|}
\hline \multicolumn{6}{|c|}{$D=0.4 \mathrm{~mm}, H=6 \mathrm{~mm}, H / D=15, \rho=1088 \mathrm{~kg} \mathrm{~m}^{-3}, \eta=\frac{1}{2} \alpha_{4}$} \\
\hline & & \multicolumn{2}{|c|}{ Nematic LCP fluid } \\
\hline$U$ & $R e$ & $F r$ & $K$ & $E r$ \\
\hline $0.0285 \mathrm{~ms}^{-1}$ & 0.3 & 0.455 & $9.4 \mathrm{E}-8$ & 5 \\
\hline $0.057 \mathrm{~ms}^{-1}$ & 0.6 & 0.91 & $1.88 \mathrm{E}-7$ & 5 \\
\hline
\end{tabular}




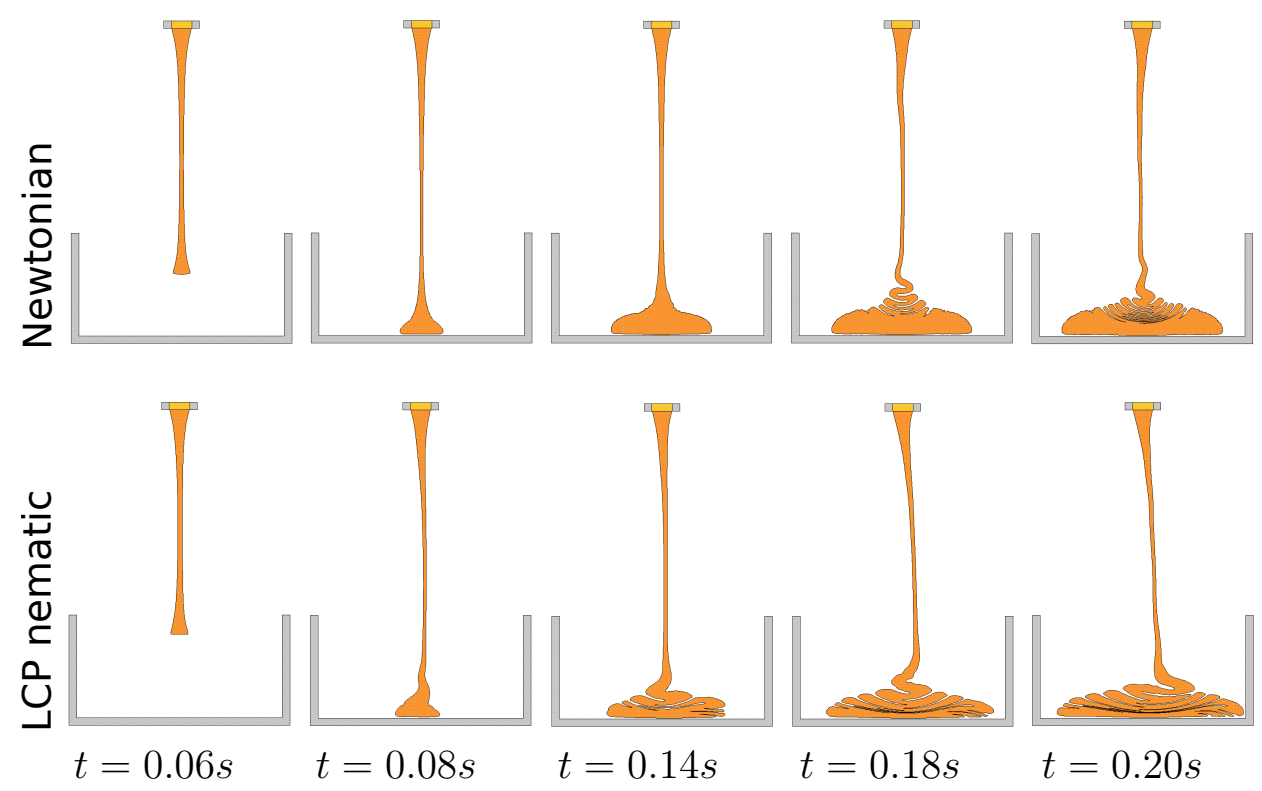

Figure 8. Numerical simulation of a jet flowing onto a rigid surface of Newtonian and nematic LCP fluids with $R e=0.3$ and $E r=5$. Fluid flow visualization at selected times.

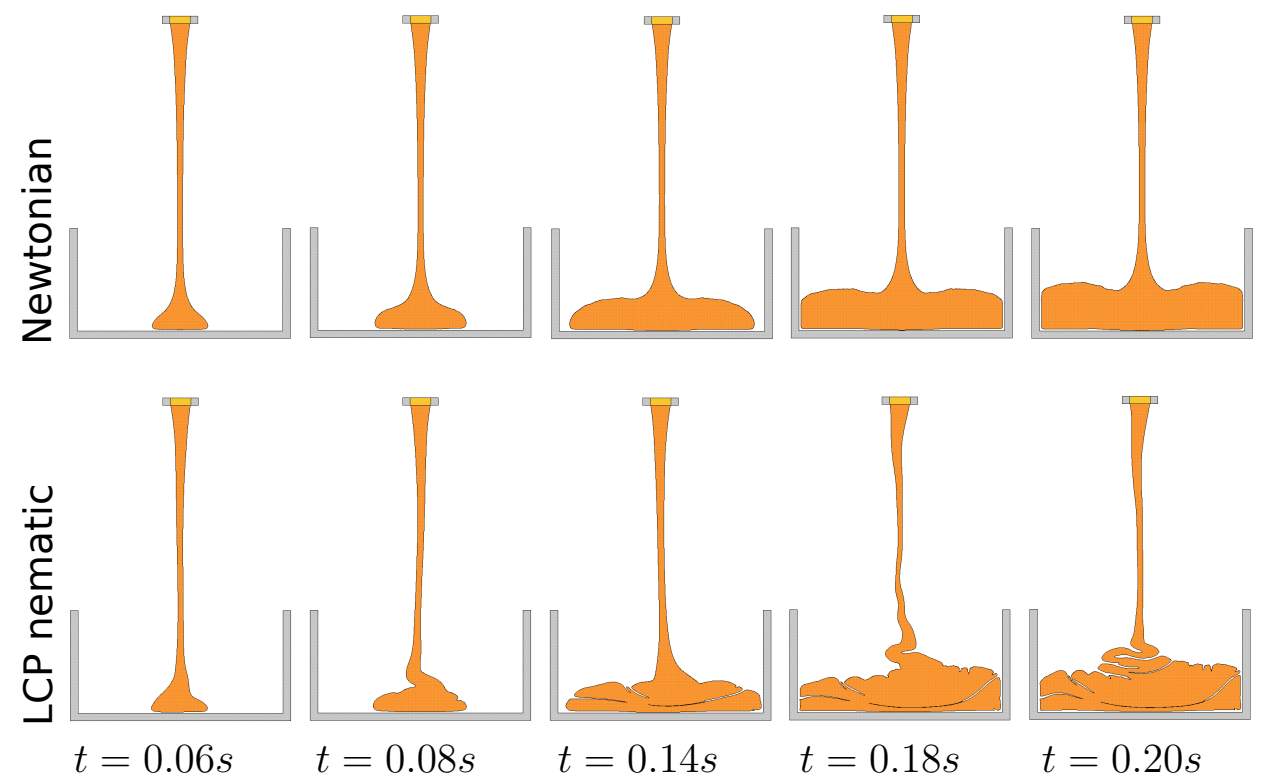

Figure 9. Numerical simulation of a jet flowing onto a rigid surface of Newtonian and nematic LCP fluids with $R e=0.6$ and $E r=5$. Fluid flow visualization at selected times. 


\subsection{The effect of the parameter $K$ on jet buckling}

To demonstrate that jet buckling is strongly affected by the parameter $K$ we show that for high values of $K$, the Ericksen-Leslie model can produce buckling results similar to the Newtonian case.

With $R e=0.6$ and $F r=0.91$, we carried out four simulations with the data shown in Table 5. Figure 10 exhibits the results obtained at times $t=0.07 \mathrm{~s}$, $0.10 \mathrm{~s}$ and $0.14 \mathrm{~s}$. The first simulation shows a Newtonian jet impinging on the bottom of the container while the second displays a nematic LCP jet for Ericksen number $E r=0.05$. To demonstrate that the elastic parameter $K$ can affect this problem, three more simulations using $E r=10,20$ and 50 were performed. As shown in Fig. 10, the differences between the results of these simulations are quite dramatic. The Cruickshank and Munson's conditions are not satisfied and the Newtonian jet did not buckle, as anticipated. The results from the simulation using $E r=0.05$ display a flow behaviour similar to the Newtonian case, while in the simulations with $E r=10,20$ and 50 the jets buckled. These results indicate that there is a greater propensity for nematic liquid crystalline polymers to buckle as the effect of the viscoelasticity diminishes, that is, as $K$ increases.

Table 5

Data employed in the simulation of jet buckling study varying $K$.

\begin{tabular}{c|c}
\hline$D=0.4 \mathrm{~mm}, H=6 \mathrm{~mm}, H / D=15, \delta x=\delta y=0.05 \mathrm{~mm}$ \\
$U=0.057 \mathrm{~ms}^{-1}, \rho=1088 \mathrm{~kg} \mathrm{~m}^{-3}, \eta=\frac{1}{2} \alpha_{4}$ \\
\hline$K$ & $E r$ \\
\hline $1.884 \mathrm{E}-5$ & 0.05 \\
\hline $9.420 \mathrm{E}-8$ & 10 \\
\hline $4.710 \mathrm{E}-8$ & 20 \\
\hline $1.884 \mathrm{E}-8$ & 50 \\
\hline
\end{tabular}



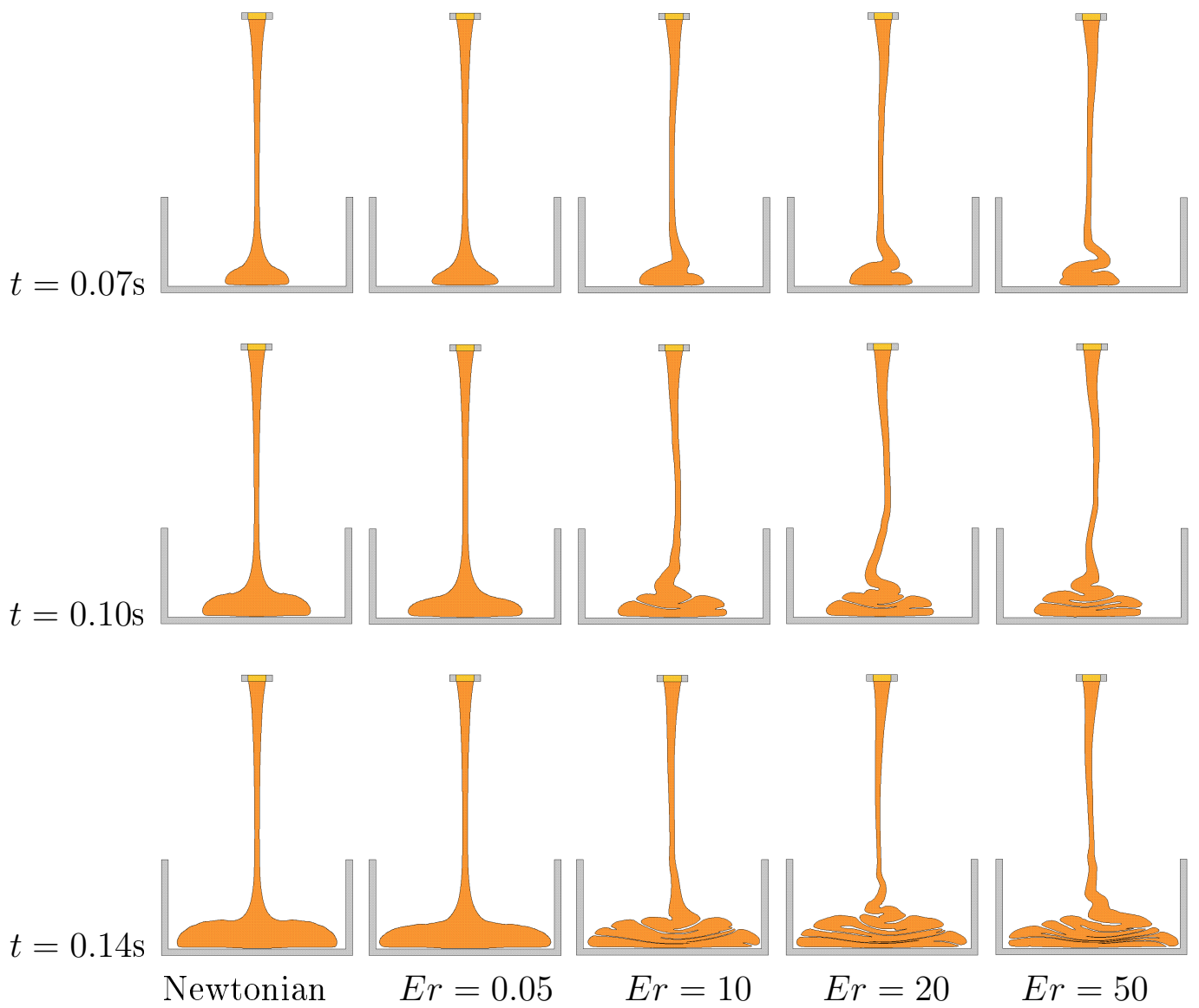

Figure 10. Numerical simulation of a jet flowing onto a rigid surface of LCP Nematic fluids with $R e=0.6$. Fluid flow visualization.

To confirm that the results obtained with $R e=0.6$ were due to elastic effects, additional simulations were performed using the elastic constant $K=$ $0.0000001569 \mathrm{~N}, U=0.095 \mathrm{~ms}^{-1}$; the values of $\delta x, \delta y, \rho, \eta$ and $D$ are given in Table 5, resulting in $R e=1, F r=1.51$ and $E r=10$. Also, corresponding simulations were performed with a Newtonian jet with $R e=1$ which does not satisfy the Cruickshank and Munson's conditions for jet buckling. Indeed, as can be seen in Fig. 11, the Newtonian jet was stable without any buckling while the nematic LCP jet was unstable displaying the buckling phenomenon. Again, these results tend to confirm that elasticity, represented by the parameter $K$ of the Ericksen-Leslie Model, can lead to jet buckling while a Newtonian one does not. 


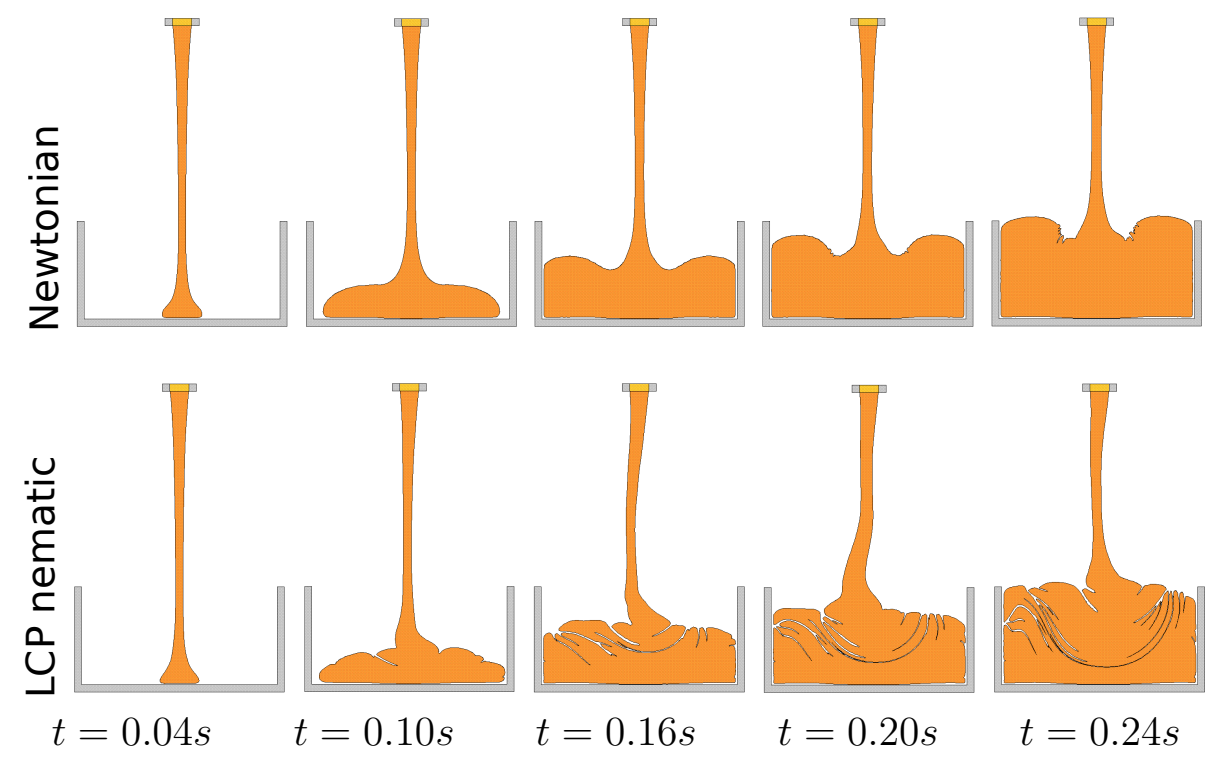

Figure 11. Numerical simulation of a jet flowing onto a rigid surface of Newtonian and LCP Nematic fluids with $R e=1$ and $E r=10$. Fluid flow visualization at selected times. 


\section{Numerical simulation of injection molding with nematic LCPs}

The simulations presented below focuses on LCPs flows during injection molding into thin-walled molds. These flow fields have one dimension ( $z$-direction) that is much smaller in comparison with the other two dimensions ( $x$ and $y$ directions), so they can be approximated as two-dimensional shear flows. The properties of the nematic fluid employed are displayed in Table 2.

In the next two subsections we present the results obtained from the simulation of the filling of the two molds, Mold A and Mold B, which are depicted in Fig. 13. The simulations were performed assuming that the molds were totally empty of air so that the initial pressure inside the molds was $p=0$. In practice, molds tend to have weep holes which would allow any air to escape.

\subsection{Mesh independency test}

To demonstrate the convergence of the numerical method for this problem, we considered Mold A and performed a mesh refinement study using the input data: $L=5 \mathrm{~mm}$ and $U=0.0075 \mathrm{~ms}^{-1}$, together with the data defining the LCP MBBA given in Table 2. With these data, we have $R e=1.0\left(\eta=\alpha_{4} / 2\right.$ was used) and $E r=1.0$ and gravity was not considered $(g=0.0)$. The meshes employed are defined in the Table 7.1 below. A total of four simulations were carried out until time $t_{\text {final }}=25.0$. Figure 12 displays the free surface profiles obtained from these simulations at times (shown in Fig. 12) $t=5,9,15,25$. By analysing Fig. 12, one concludes that the solutions obtained on meshes M0, M1 and M2 converge to that of mesh M5 which shows that the method is convergent.

Table 6

Meshes employed in the mesh refinement study of injection molding of Mold A.

\begin{tabular}{|c|c|c|c|c|}
\hline Meshes & M0 & M1 & M2 & M3 \\
\hline$\delta x=\delta y$ & $\mathrm{~L} / 8$ & $\mathrm{~L} / 10$ & $\mathrm{~L} / 16$ & $\mathrm{~L} / 20$ \\
\hline Mesh sizes & $68 \times 56$ & $86 \times 70$ & $136 \times 112$ & $172 \times 140$ \\
\hline
\end{tabular}


(a) $t=5$

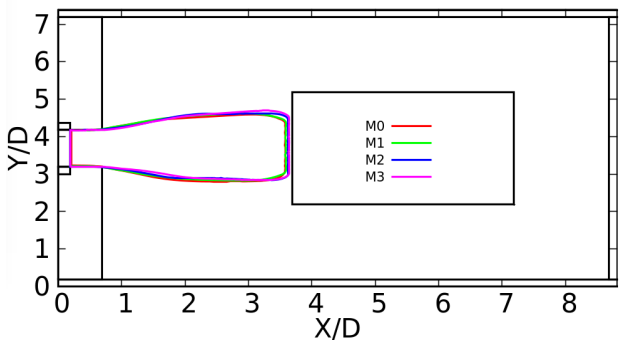

(c) $\mathrm{t}=15$

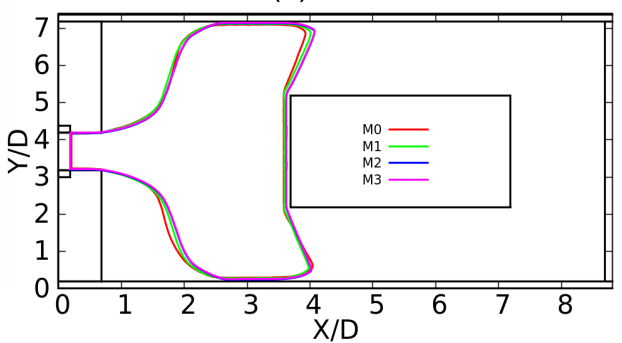

(b) $t=9$

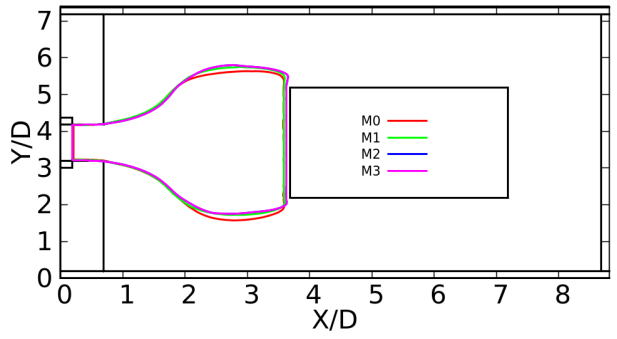

(d) $t=25$

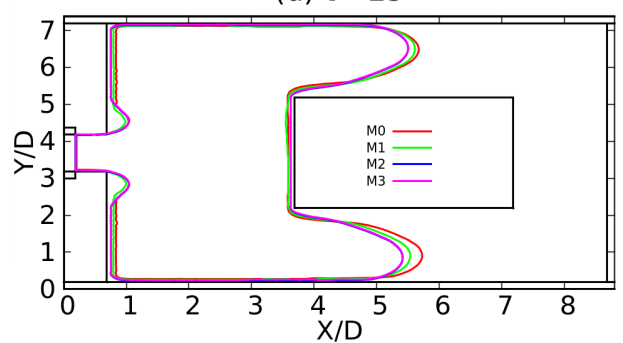

Figure 12. Mesh refinement study using $R e=1$ and $E r=1$. Free surface profiles obtained on meshes M0, M1, M2 and M3 at selected times shown. 
a) Mold A

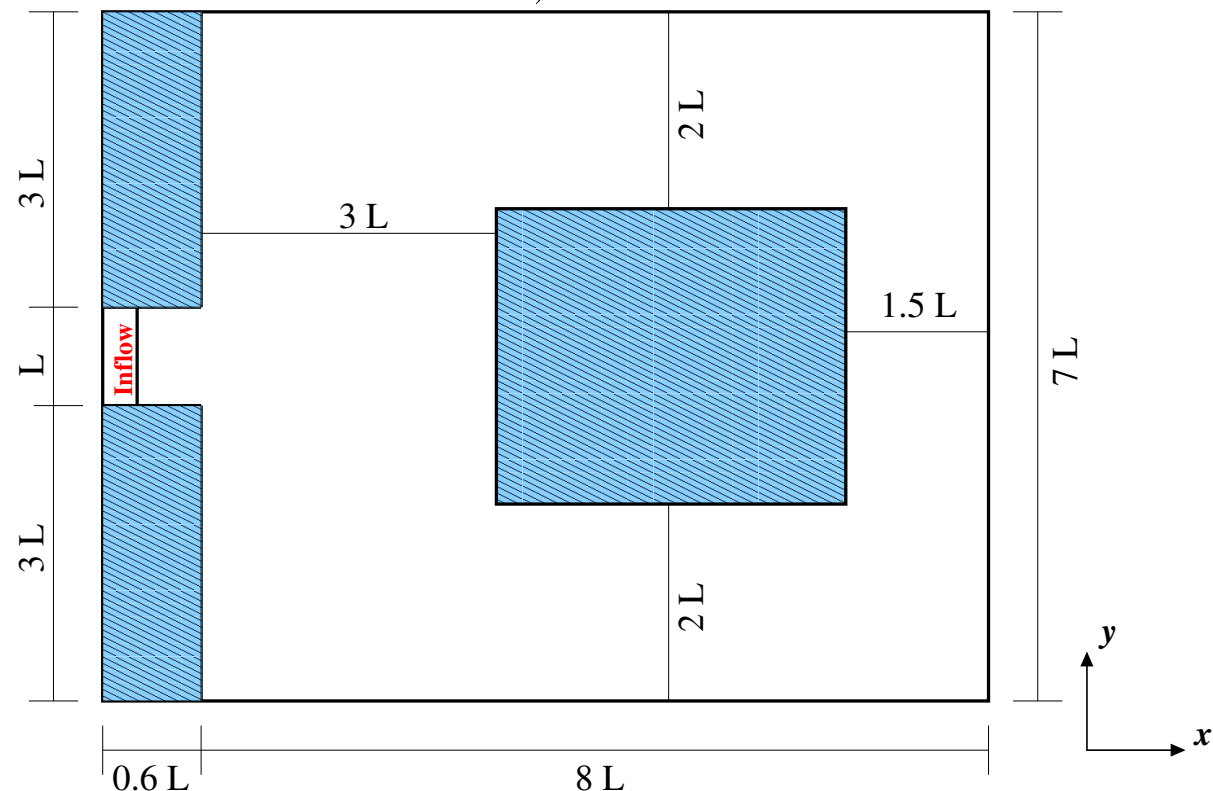

b) Mold B

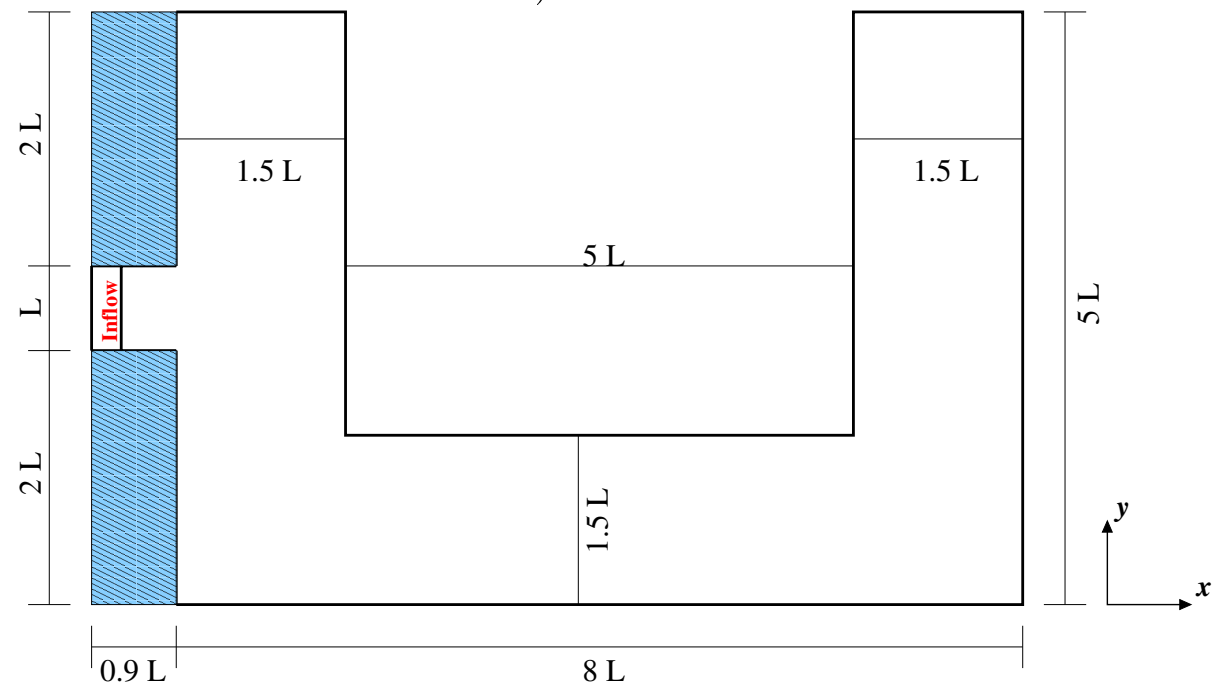

Figure 13. Mold geometries employed. 


\subsection{Simulation of the filling of Mold A}

We present the results obtained in the simulation of the filling of Mold A where a square obstacle of dimensions $3.5 \mathrm{~L} \times 3.0 \mathrm{~L}$ is placed in its interior (see Fig. 13a). The dimensions of this mold are $7 L$ by $8.5 L$, where $L$ denotes the size of the inlet injection nozzle (see Fig. 13a). The boundary conditions for the velocity field were those specified in Section 3 while, at the inflow, we employed the fully developed nematic profile, that was derived by Cruz et al. [15] for channel flow under the assumption of small director angles:

$$
\begin{aligned}
& u(y)=\frac{R e}{2\left[1+\frac{1}{2}\left(\alpha_{3}+\alpha_{6}\right)\right]} p_{, x} y(y-1), \\
& u_{, y}=\frac{R e}{2\left[1+\frac{1}{2}\left(\alpha_{3}+\alpha_{6}\right)\right]} p_{, x}(2 y-1), \\
& \phi(y)=\frac{E r}{2}\left(\gamma_{1}+\gamma_{2}\right) \frac{R e}{2\left[1+\frac{1}{2}\left(\alpha_{3}+\alpha_{6}\right)\right]} p_{, x}\left[\frac{y(1-y)(1-2 y)}{6}\right] .
\end{aligned}
$$
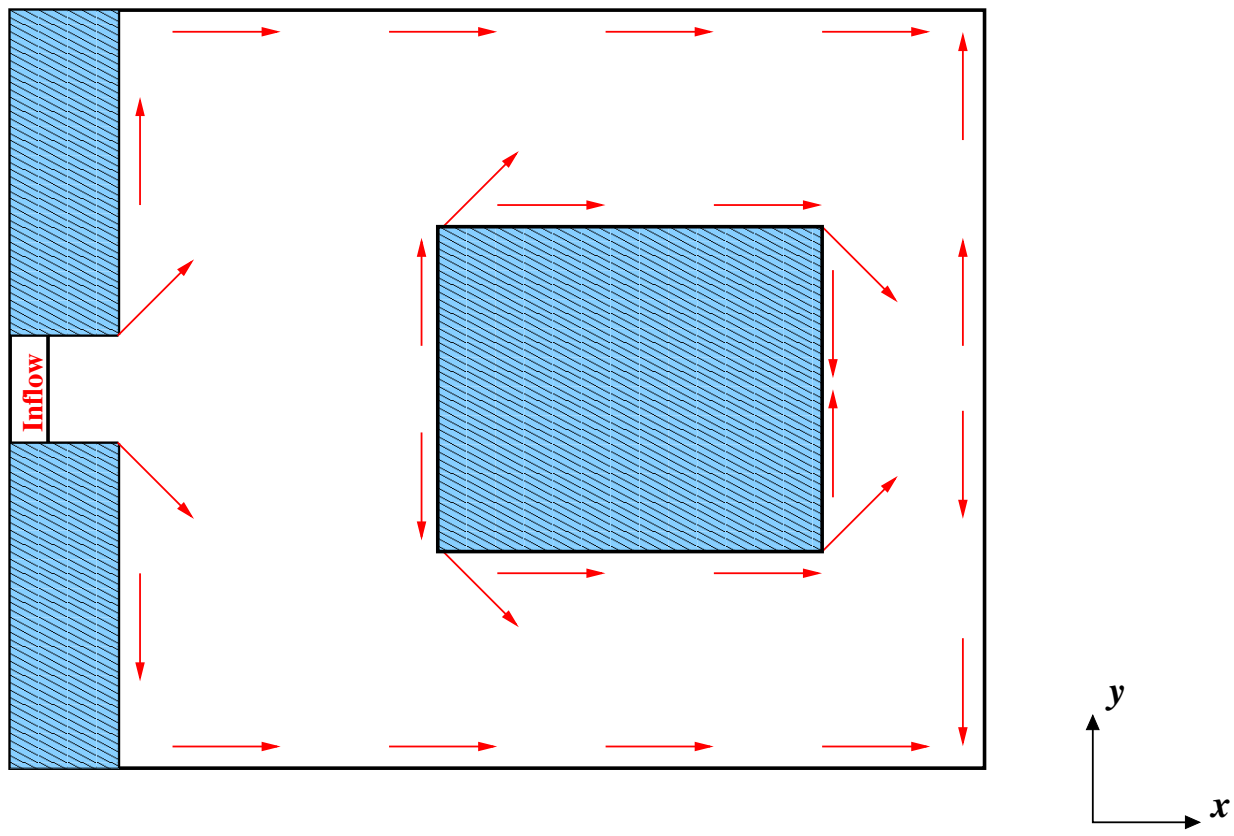

Figure 14. Definition of the boundary conditions (represented by the arrows) used for the calculation of the orientation angle $\phi$. 
The boundary conditions for the angle $\phi$ were specified as follows (see Fig. 14):

1. At the injection nozzle (entrance of the mold) $\phi=0$.

2. Along the horizontal walls, the anchoring angle was set to zero, implying parallel alignment to the walls.

3. At the vertical walls we employed:

a) $\phi=-90^{\circ}$ at $x=0.5 L$ and $0 L \leq y \leq 3 L$;

a) $\phi=90^{\circ}$ at $x=0.5 \mathrm{~L}$ and $4 L \leq y \leq 7 \mathrm{~L}$;

a) $\phi=-90^{\circ}$ at $x=3.5 \mathrm{~L}$ and $2 \mathrm{~L} \leq y \leq 3.5 \mathrm{~L}$;

b) $\phi=90^{\circ}$ at $x=3.5 \mathrm{~L}$ and $3.5 \mathrm{~L} \leq y \leq 5 \mathrm{~L}$;

c) $\phi=90^{\circ}$ at $x=7.0 \mathrm{~L}$ and $2 L \leq y \leq 3.5 \mathrm{~L}$;

d) $\phi=-90^{\circ}$ at $x=7.0 \mathrm{~L}$ and $3.5 \mathrm{~L} \leq y \leq 5 \mathrm{~L}$;

e) $\phi=-90^{\circ}$ at $x=8.5 \mathrm{~L}$ and $0 L \leq y \leq 3.5 \mathrm{~L}$;

f) $\phi=90^{\circ}$ at $x=8.5 \mathrm{~L}$ and $3.5 \mathrm{~L} \leq y \leq 7 \mathrm{~L}$.

Table 7

Data employed in the simulation of the filling of Mold A.

\begin{tabular}{|ccc|cc|}
\hline \multicolumn{4}{|l|}{$U=0.003 \mathrm{~ms}^{-1}, \rho=1088 \mathrm{Kg} \mathrm{m}^{-3}, \rho=1088 \mathrm{~kg} \mathrm{~m}^{-3}, \eta=\frac{1}{2} \alpha_{4}$} \\
\hline & & & \multicolumn{2}{|c|}{ LCP Nematic fluid } \\
\hline$L(\mathrm{~m})$ & $R e$ & $F r$ & $K(\mathrm{~N})$ & $E r$ \\
\hline 0.003 & 0.24 & 0.018 & $3.72 \mathrm{E}-7$ & 1 \\
\hline 0.005 & 0.40 & 0.014 & $6.20 \mathrm{E}-7$ & 1 \\
\hline 0.007 & 0.55 & 0.012 & $8.67 \mathrm{E}-7$ & 1 \\
\hline
\end{tabular}

The parameters specifying the flow are given by Table 7 . The simulations were performed using the following computational domain: 119 cells $\times 98$ cells with grid spacing $\delta x=\delta y=0.5 \mathrm{~mm}$. Figs. 15 and 16 display the isolines of $u$ and $v$ at times $t=40 \mathrm{~s}$ and $t=76 \mathrm{~s}$, where the length scale was $L=0.007 \mathrm{~m}$ giving $R e=0.55$ and $E r=1$. Although not shown explicitly, the mold (e.g. Fig. 14 ) is assumed to have weep holes which allow air to escape. Since the flow is relatively slow, it is not unreasonable to assume that any air within the mold is at atmospheric pressure (i.e. $p=0$ ).

Figure 17 presents the locations of the free surface at various times during the filling operations of Mold A for the three sizes of the cross-section of the injection inlet nozzle. The filling time for $L=0.003 \mathrm{~m}$ was approximately 178 seconds while for $L=0.005 \mathrm{~m}$ and $L=0.007 \mathrm{~m}$ the filling times were $107 \mathrm{~s}$ and $76 \mathrm{~s}$, respectively. 

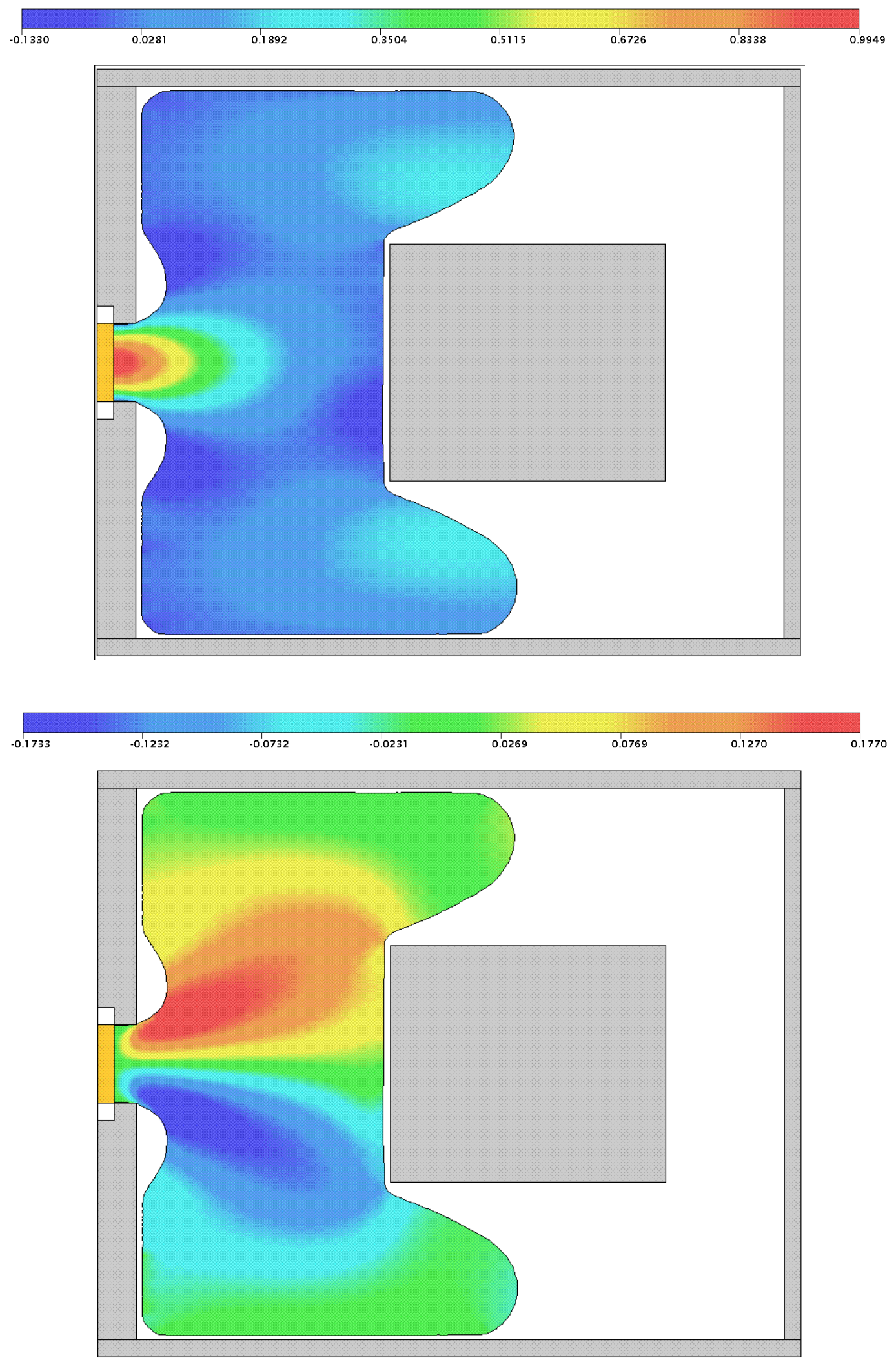

Figure 15. Numerical simulation of the filling of Mold A with $L=7 \mathrm{~mm}, R e=0.55$ and $E r=1$. Isolines of $u$ and $v$ at time $t=40$ s. 

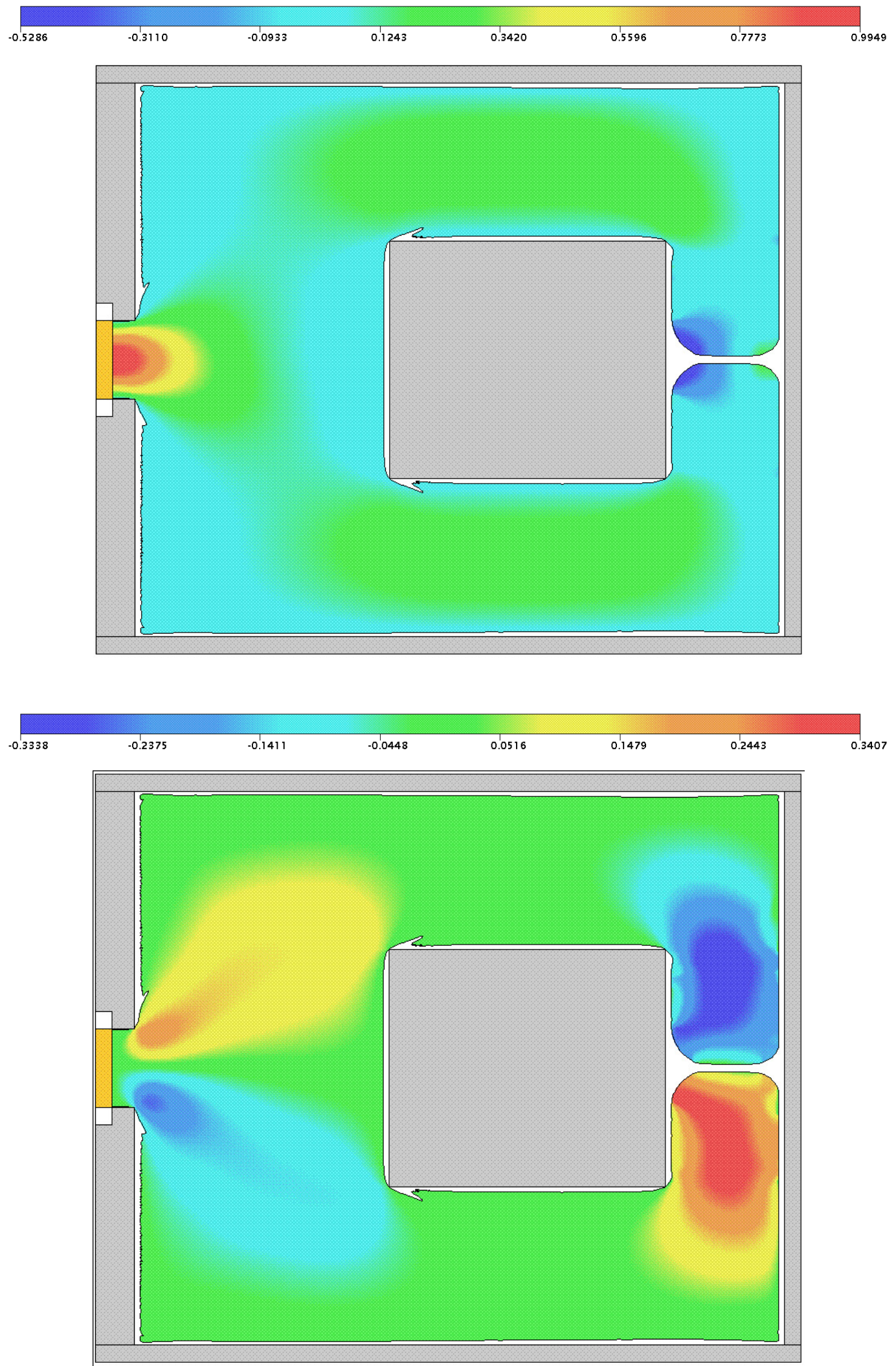

Figure 16. Numerical simulation of the filling of Mold A with $R e=0.55$ and $E r=1$. Isolines of $u$ (above) and $v$ (below) at time $t=76 \mathrm{~s}$. 


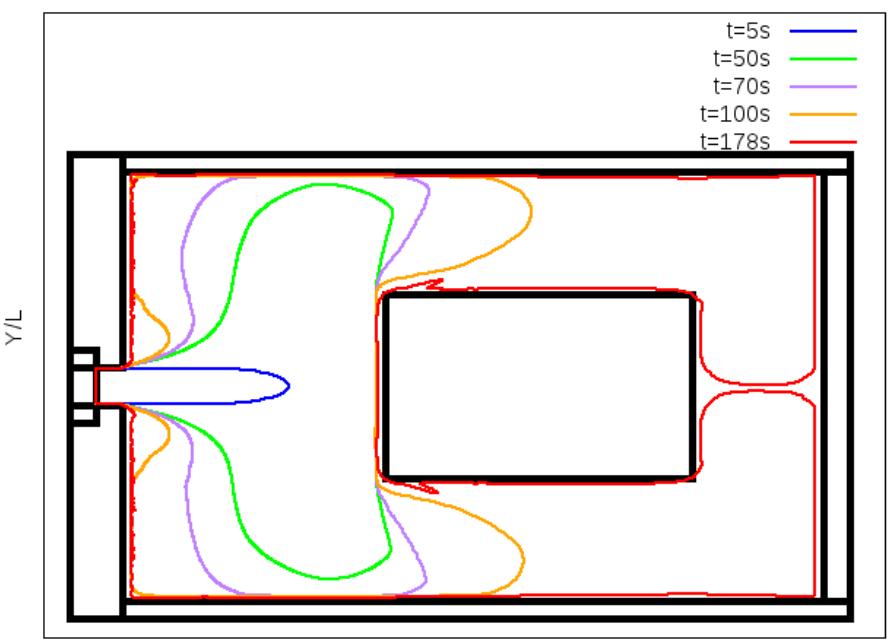

a) $\mathrm{L}_{1}=0.003 \mathrm{~m}$

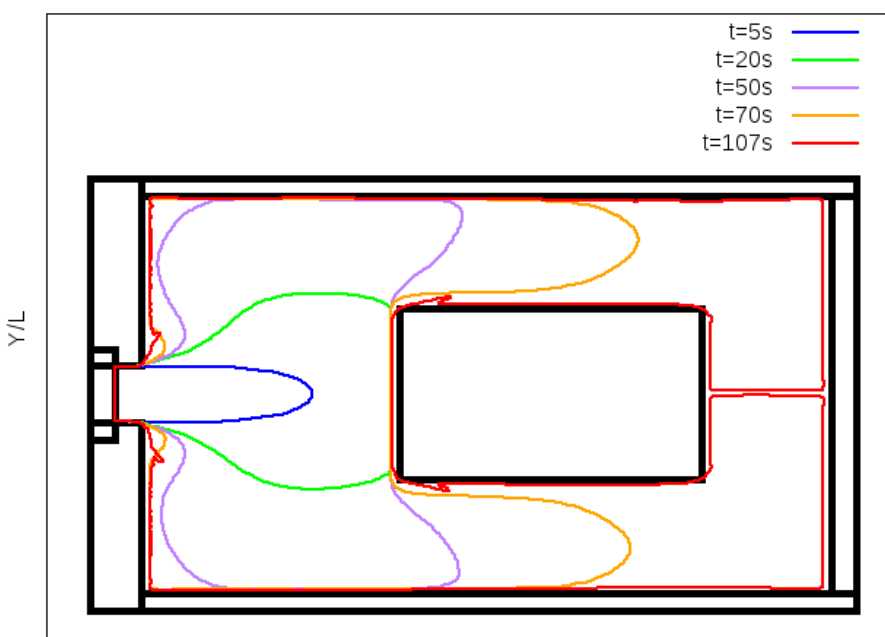

b) $\mathrm{L}_{2}=0.005 \mathrm{~m}$

XL

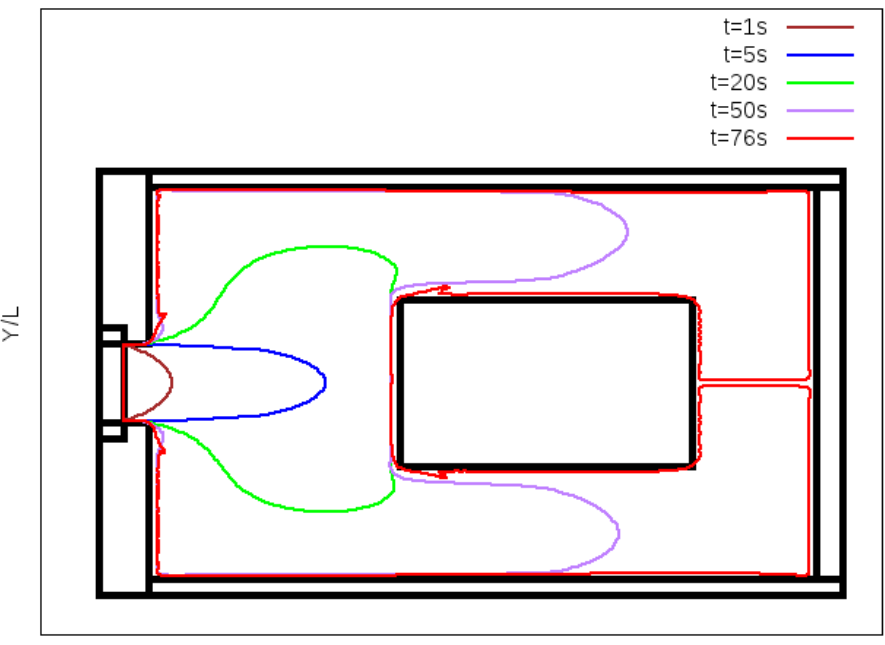

c) $\mathrm{L}_{3}=0.007 \mathrm{~m}$

Figure 17. Development of the free surface using three sizes of injection points $(L=3,5,7 \mathrm{~mm})$. 
The filling time ( $t_{f}$ in seconds) would appear to be inversely proportional to the square of the cross-section of the inlet nozzle ( $L$ in S.I. units). This relation can be approximated by the equation

$$
t_{f}=\frac{c}{L^{2}},
$$

where $c$ is a constant that was determined by the least squares method which gave $c \approx 0.002$. The predicted filling time, as a function of the cross-section of the injection nozzle is given in Fig. 18 .

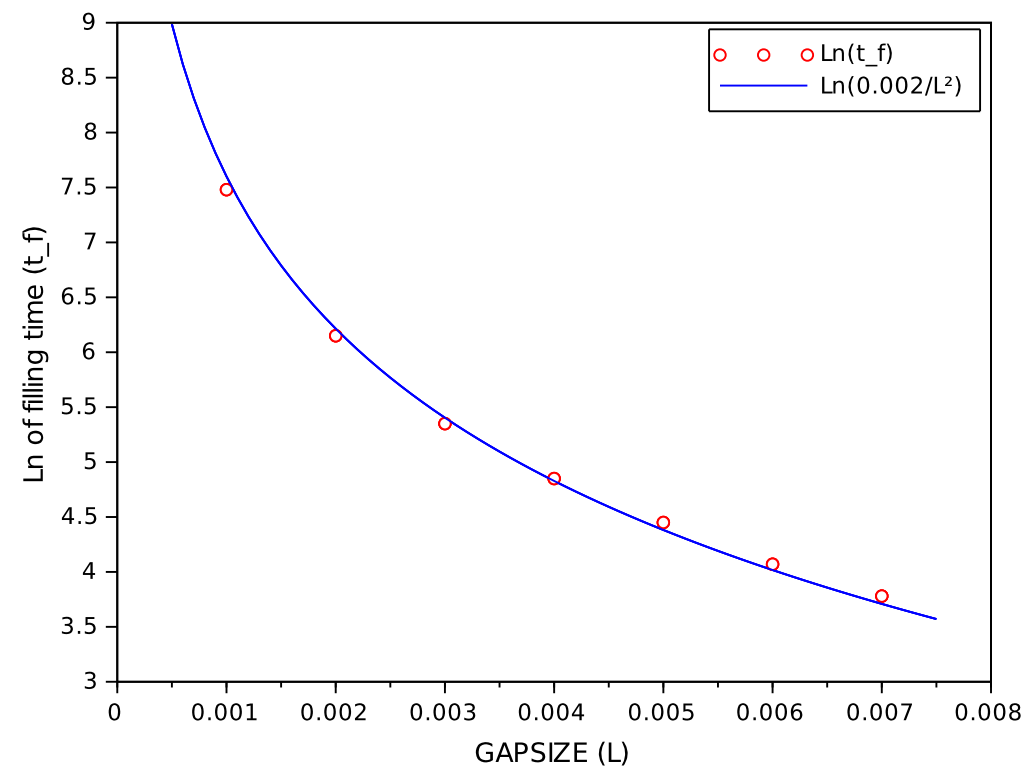

Figure 18. Prediction of the filling time $\left(t_{f}\right)$ as function of the cross-section of the inlet nozzle $(L)$.

Figure 19 displays the flow directions for $L=0.005 \mathrm{~m}$ near the completion of the filling stage. It can be seen that the velocity field generally agrees with the direction of the moving free surface and no recirculation is observed. A similar result was obtained with the other nozzle sizes $(L)$. A possible weld line is visible where the two fluid fronts meet in the right vertical channel.

The distribution of the director orientations are displayed in Figs. 20, 21 and 22 where two zoom-in zones are shown. The three different inlet nozzle sizes do not have a great effect on the director orientation, although it is noticeable that for the larger nozzle (Fig. 22) the director on the left wall, above and below the nozzle inlet, are tilted at, respectively, an angle of approximately $135^{\circ}$ and $-135^{\circ}$, whereas those in the smaller nozzle (Fig. 20) lie between $45^{\circ}$ and $90^{\circ}$ (above the nozzle) and $270^{\circ}$ (below the nozzle) and are shifted more to the left. 


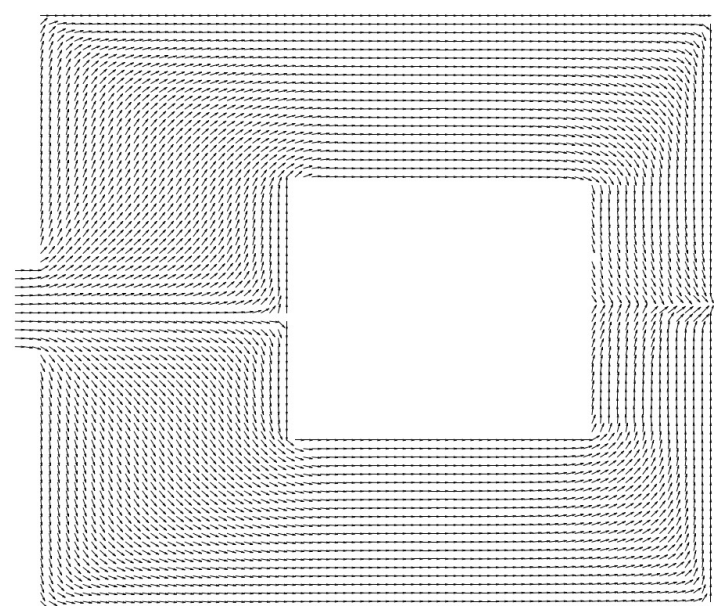

Figure 19. Velocity vectors obtained in the simulation of the filling of Mold A at time $t=76 \mathrm{~s}$.

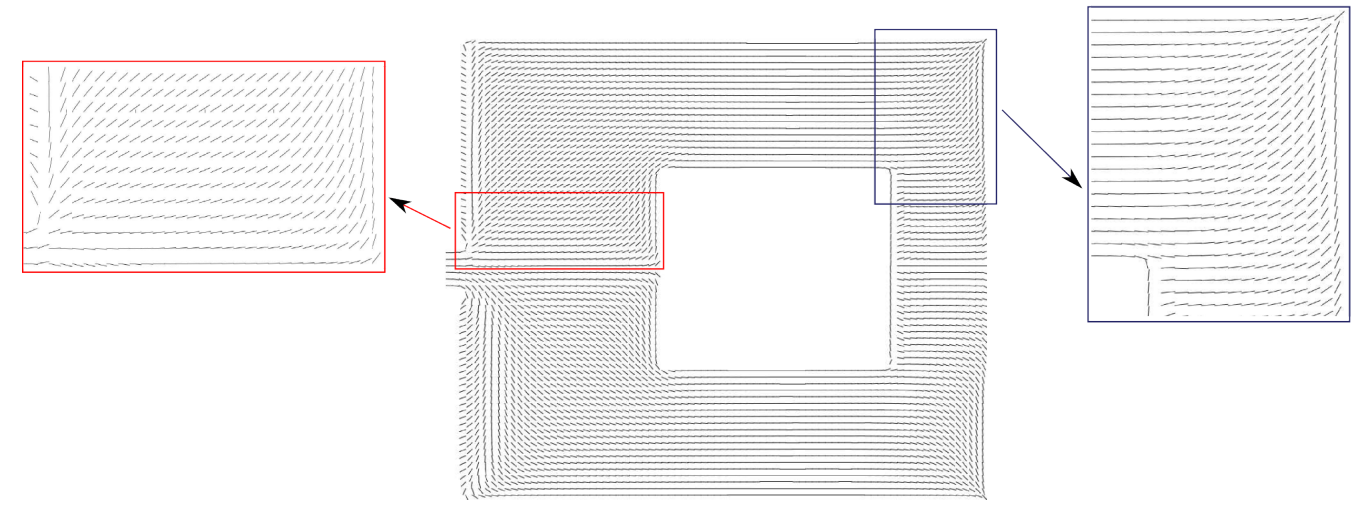

Figure 20. Director orientations in the simulation of the filling of Mold A. Inlet cross-section $=0.003 \mathrm{~m}$.

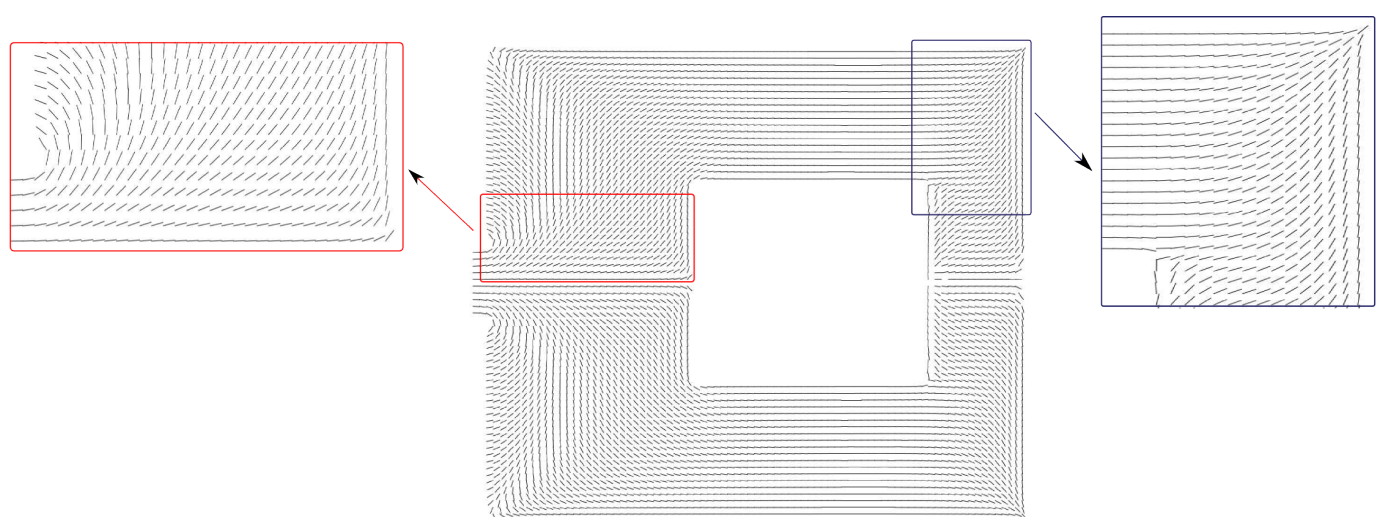

Figure 21. Director orientations in the simulation of the filling of Mold A. Inlet cross-section $=0.005 \mathrm{~m}$. 

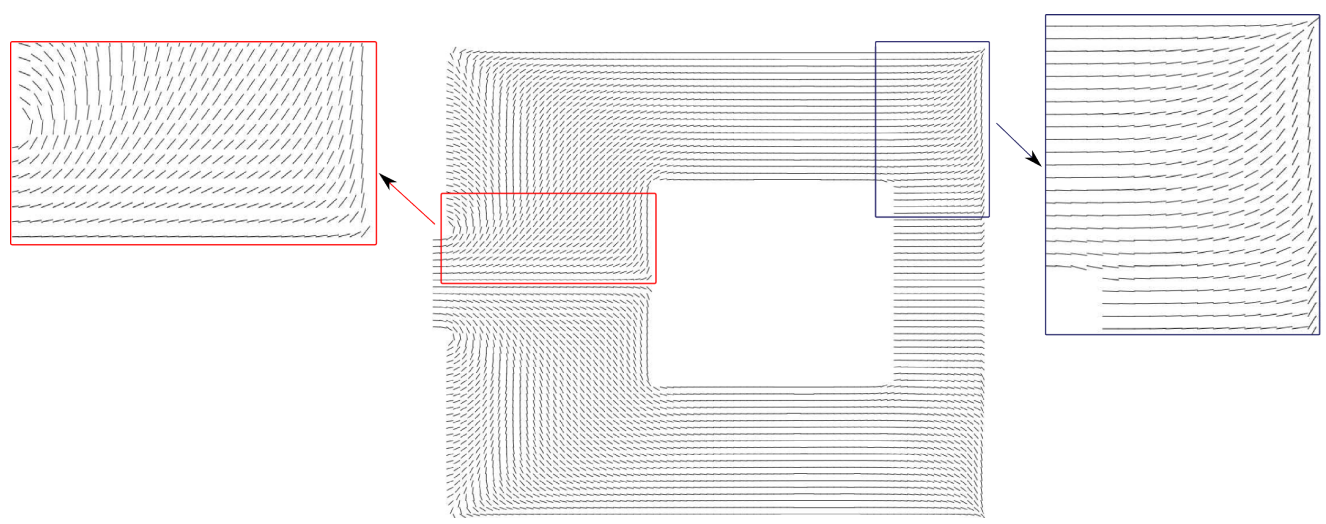

Figure 22. Director orientations in the simulation of the filling of Mold A. Inlet cross-section $=0.007 \mathrm{~m}$.

\subsection{Numerical simulation of the filling of Mold B.}

We applied the method of Section 4 to simulate the filling of Mold B as shown in Fig. 13(b). The boundary conditions for the velocity field are described in Section 3 while at the channel entrance the $x$-component of the velocity was given by the analytic expression (24a).

The following boundary conditions were specified (see also Fig. 23):

1. Along the horizontal walls, the anchoring angle was set to zero, implying parallel alignment to the walls.

2. At the contraction entrances, we used:
a) $(\mathrm{BC} 1$ and $\mathrm{BC} 2) \phi=-45^{\circ}$;
b) (BC3) $\phi=45^{\circ}$;

3. At the expansion entrances, we used:
a) $\phi=-45^{\circ}$;
b) $\phi=45^{\circ}$;

4. At the vertical walls (i e, the contraction walls and final walls), we employed: BC1) $\phi=-90^{\circ}$ at $x=2.4 \mathrm{~L}$ and $1.5 \mathrm{~L} \leq y \leq 2.5 \mathrm{~L}$, and $\phi=90^{\circ}$ at $x=2.4 \mathrm{~L}$ and $2.5 L \leq y \leq 5 L$

BC2) $\phi=-90^{\circ}$ at $x=2.4 \mathrm{~L}$ and $1.5 \mathrm{~L} \leq y \leq 5 \mathrm{~L}$;

BC3) $\phi=90^{\circ}$ at $x=2.4 \mathrm{~L}$ and $1.5 \mathrm{~L} \leq y \leq 5 \mathrm{~L}$; $\phi=90^{\circ}$ at $x=8.9 \mathrm{~L}$ and $0 \mathrm{~L} \leq y \leq 5 \mathrm{~L}$;

5. At the vertical walls (or the expansion walls), we employed:

a) $\phi=-90^{\circ}$ at $x=0.9 \mathrm{~L}$ and $0 \leq y \leq 2 \mathrm{~L}$;

b) $\phi=90^{\circ}$ at $x=0.9 L$ and $3 L \leq y \leq 5 L$;

b) $\phi=90^{\circ}$ at $x=7.4 \mathrm{~L}$ and $1.5 \mathrm{~L} \leq y \leq 5 \mathrm{~L}$;

6. At the channel entry defined by $x=0$ and $2 L \leq y \leq 3 L$, we set $\phi=0$.

The physical parameters, specific to this nematic LCP problem, are presented in Table 2. The remaining input parameters are given in Table 8. The simulations were performed using the following computational domain $4.45 \mathrm{~mm} \times$ 
$2.5 \mathrm{~mm}$ with grid spacing $\delta x=\delta y=0.5 \mathrm{~mm}$.

Table 8

Data employed in the simulation of the filling of Mold B.

\begin{tabular}{ccc|cc}
\hline \multicolumn{4}{l}{$U=0.005 \mathrm{~ms}^{-1}, \rho=1088 \mathrm{Kg} \mathrm{m}^{-3}, \eta=\frac{1}{2} \alpha_{4}$ Pa.s } \\
\hline$L(\mathrm{~m})$ & $R e$ & $F r$ & $K(\mathrm{~N})$ & $E r$ \\
\hline 0.005 & 0.66 & 0.023 & $1.03 \mathrm{E}-6$ & 1 \\
\hline
\end{tabular}

To demonstrate that the boundary conditions on the orientation angle $\phi$ can affect the director and the velocity fields, three simulations using $R e=0.7$ and $E r=1$ were performed. In the first simulation we used the boundary conditions BC1 displayed in Fig. 23(a) while in the second and third simulations the boundary conditions were $\mathrm{BC} 2$ and $\mathrm{BC} 3$ described in Figs. 23(b) and 23(c), respectively. The solutions obtained for the director orientation are shown in Fig. 25.

It is seen that the particular angle of the anchored director on the boundary has an effect on the flow. If we examine Fig. 24 and focus on the free surface at $t=12 \mathrm{~s}$, then we see a distinct difference between the three diagrams. Clearly BC3 with its anchoring on the right side wall of the first vertical channel all in the positive $y$-direction is the slowest, while $\mathrm{BC} 2$ with its anchoring all in the negative $y$-direction is the fastest. The internal orientation of the director in the first vertical channel (see Fig. 25) is also markedly different in the three cases. The difference between $\mathrm{BC} 2$ and $\mathrm{BC} 3$ is the most noticeable just after the fluid emerges from the nozzle where the orientation of the directors differ by approximately $90^{\circ}$. 
a) $\mathrm{BC1}$
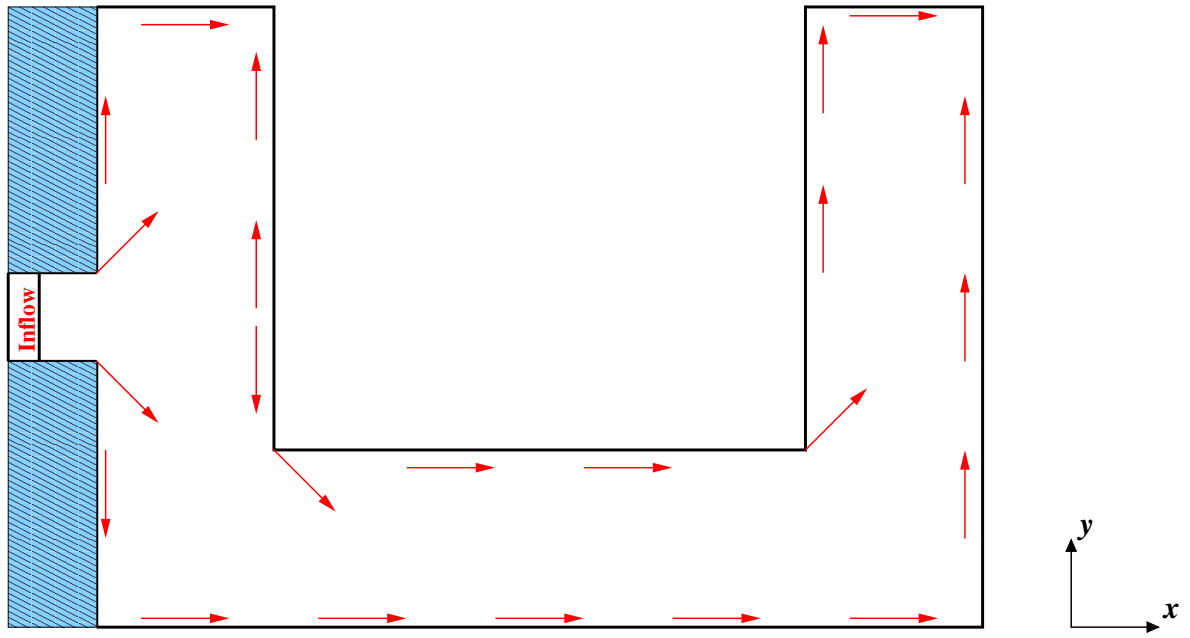

b) $\mathrm{BC} 2$
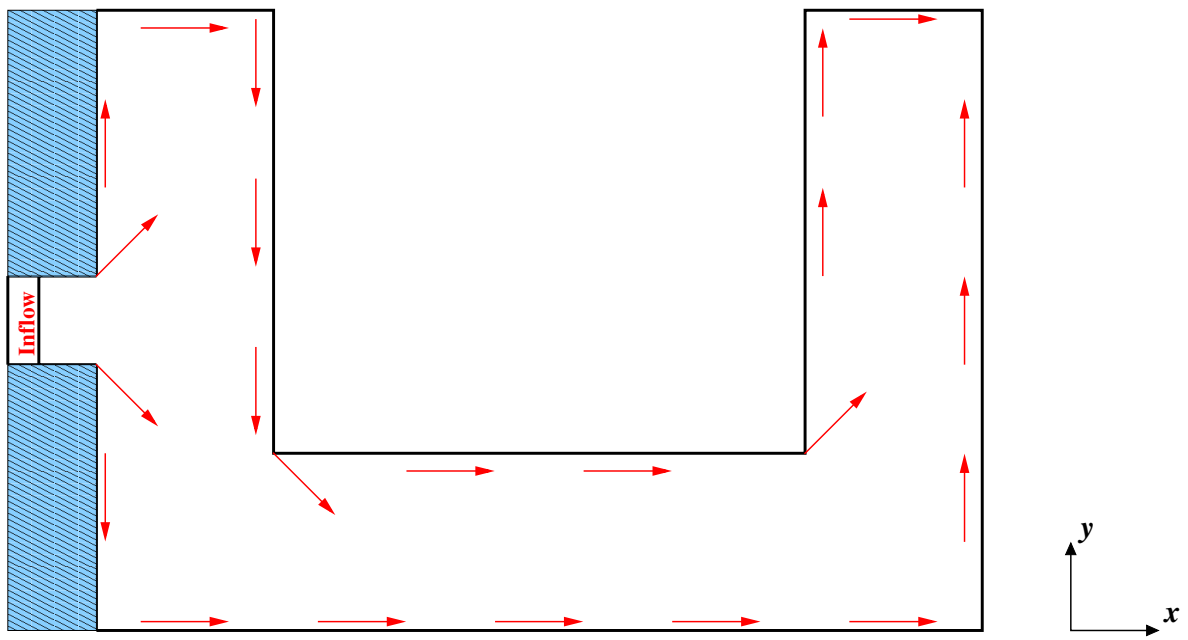

c) $\mathrm{BC3}$
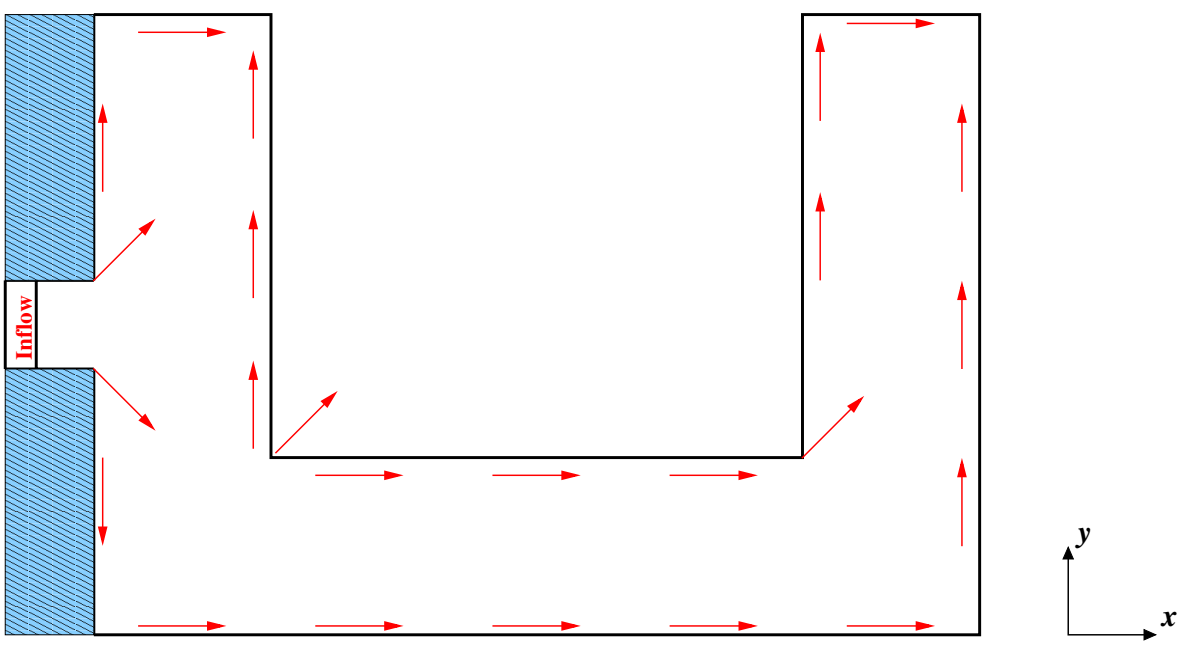

Figure 23. Description of the domain for the simulation of the flow. The red arrows represent the boundary conditions used for the calculation of the angle $\phi$. 
a) $\mathrm{BC1}$

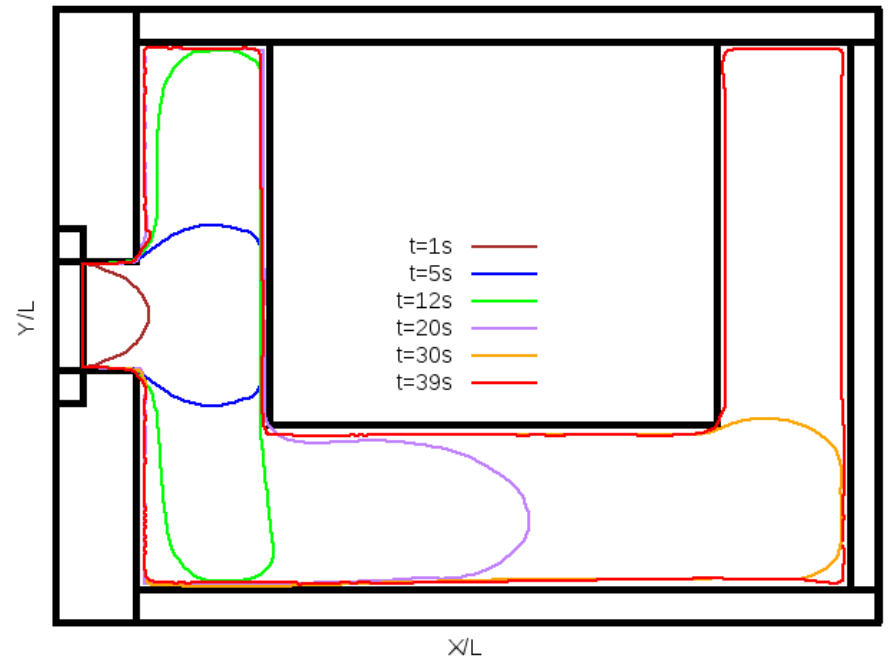

b) $\mathrm{BC} 2$

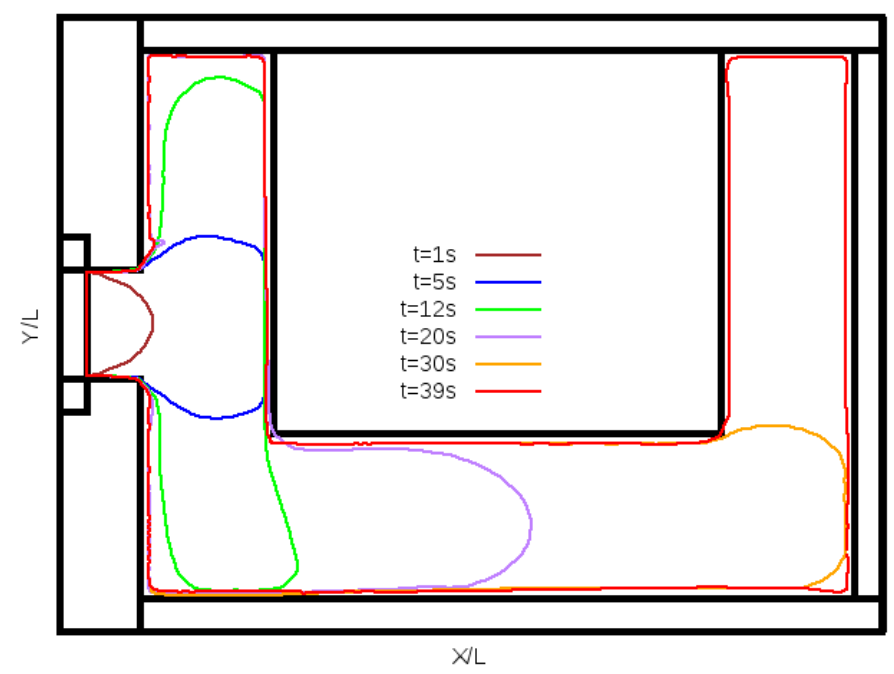

c) $\mathrm{BC3}$

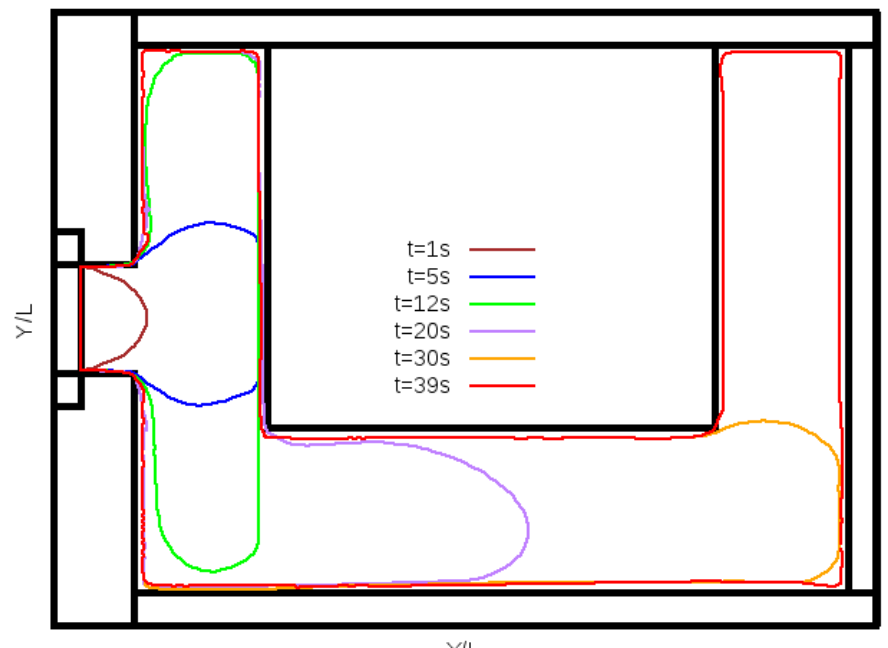

Figure 24. Development of free surface at selected times for different boundary conditions on the angle $\phi$. 
a) $\mathrm{BC1}$

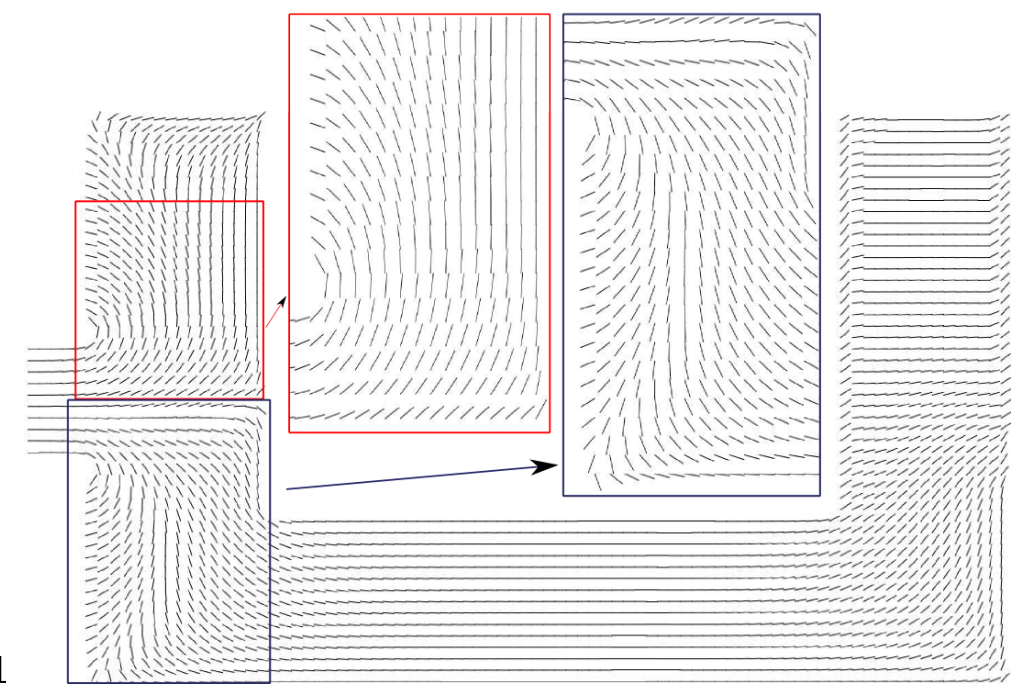

b) $\mathrm{BC} 2$

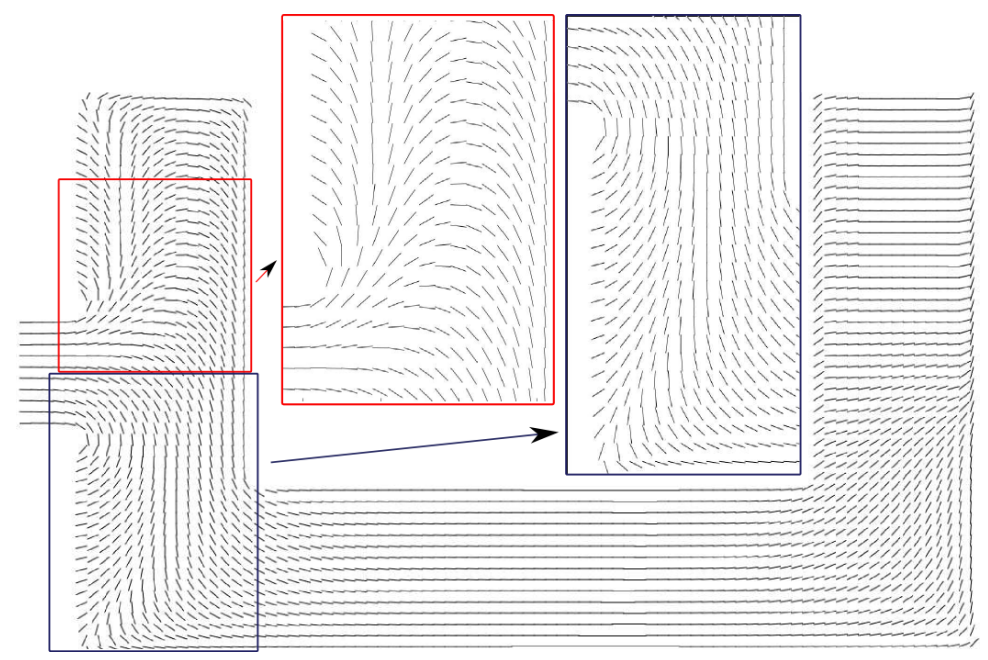

\section{c) $\mathrm{BC} 3$}

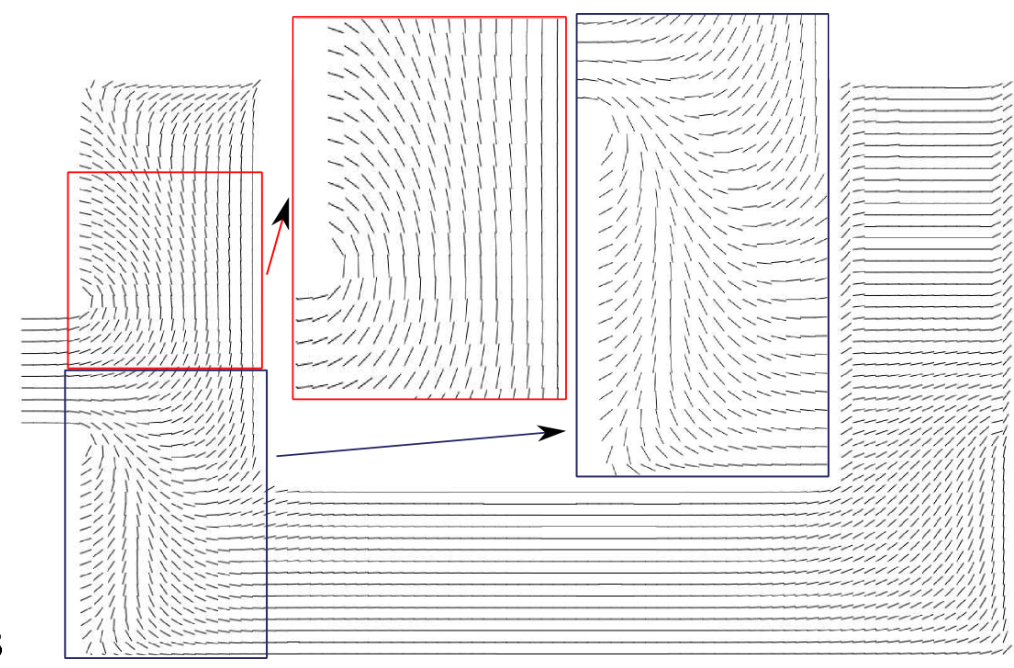

Figure 25. Director orientations in the simulation of the filling of Mold B for different boundary conditions on the angle $\phi$. 


\section{Concluding Remarks}

This paper has been concerned with the development of a numerical technique for solving free surface flows of liquid crystalline polymers described by the Ericksen-Leslie dynamic equations. The governing equations were written down and a discussion of the boundary conditions on both rigid and free surfaces boundaries then followed. The finite difference algorithm was based on the marker-and-cell (MAC) philosophy and developed from the ideas of the earlier work of Cruz et al. [15] for confined flows. Several improvements to this paper were included: a technique for dealing with moving free surfaces was implemented; the momentum equations were solved by the implicit Euler method and the pressure condition on the free surface was computed implicitly.

Numerical verification of the method was indicated by running the code using several mesh sizes demonstrating that it was capable of solving free surface flows governed by the Ericksen-Leslie dynamic equations. The problem of a jet impinging on a rigid surface was simulated for various values of Ericksen numbers. The results showed the occurrence of the phenomenon of jet buckling over a range of viscoelastic polymer fluids. This was in contrast to Newtonian fluids where both the experimental and theoretical work of Cruickshank and Munson provided a good indication as to when buckling would occur. Injection molding of liquid crystalline polymers were also studied. The simulations predicted the location of the melt front during the injection process. The director orientation on the boundaries were seen to have a substantial influence on the flow and the director orientation within the fluid. Finally, we observed that the filling time, at least for the aspect ratio of the particular mold, was inversely proportional to the square of the entrance nozzle width.

\section{ACKNOWLEDGEMENTS}

The Brazilian authors would like to acknowledge the financial support given by the Brazilian funding agencies: $\mathrm{CNPq}$ - Conselho Nacional de Desenvolvimento Científico e Tecnológico, Grants Nos. 136974/2014-7, 457373/2014-8, No. 306280/2014-0, FAPESP - Fundação de Amparo a pesquisa do Estado de São Paulo, Grants No. 2013/07375-0) (CEPID-CeMEAI project), The last author (S. McKee) would like to acknowledge a travel grant from the Royal Society of Edinburgh. 


\section{References}

[1] M. Alves, P. Oliveira, F. Pinho, A convergent and universally bounded interpolation scheme for the treatment of advection, Int. J. Num. Meth. Fluids, 41 (2003), 47-75.

[2] C. Atkinson, P. J. S. Pereira, Flow of a nematic liquid crystal near the leading edge of an infinite prism, Q. J. Mech. Appl. Math., 58 (2005), 503-533.

[3] J. N. Baleo, M. Vincent, P. Navard, Finite-element simulation of flow and director orientation of viscous anisotropic fluids in complex 2D geometries, $J$. Rheol., 36(1) (1992), 663-701.

[4] G. K. Batchelor, An Introduction to Fluid Dynamics, Cambridge University Press, Cambridge, 1967.

[5] B. Bird, R. C. Armstrong, O. Hassager. Dynamics of Polymeric Liquids, Wiley, New York, 1987, Vol. 1.

[6] J. D. Bunning, D. A. Crellin, T. E. Faber, The effect of molecular biaxiality on the bulk properties of some nematic liquid crystals, Liq. Cryst., 1 (1986), 37-51.

[7] Rong-Yeu Chang, Fu-Chia Shiao, Wen-Lii Yang, Simulation of director orientation of liquid crystalline polymers in 2-D flows, J. Non-Newtonian Fluid Mech., 55 (1994), 1-20.

[8] C. L. Choy, W. P. Leung, K. W. Kwok, Thermal diffusivity of an injectionmoulded liquid crystalline polymer by flash radiometry, Polym. Commun., 32 (1991), 285-288.

[9] S. Chono, T. Tsuji, Numerical simulation of nematic liquid crystalline flows around a circular cylinder, Mol. Liq. Cryst., 309 (1998), 217-236.

[10] S. Chono, T. Tsuji, Computer simulation of flow and defect of nematic liquid crystal. EKISHO (in Japanese), 3 (1999), 107-116.

[11] S. Chono, T. Tsuji, M. M, Denn, Spatial development of director orientation of tumbling nematic liquid crystals in pressure-driven channel flow, J. NonNewtonian Fluid Mech., 79 (1998), 515-527.

[12] A. J. Chorin, Numerical solution of the Navier-Stokes equations, J. Comput. Phys., 2 (1968), 745-762.

[13] D. K. Cinader, W. R. Burghardt, Polydomain model predictions of liquid crystalline polymer orientation in mixed shear/extensional channel flows, Rheol. Acta., 39 (2000), 259.

[14] J. O. Cruickshank, B. R. Munson, Viscous-fluid buckling of plane axisymmetric jets, J. Fluid Mech., 113 (1981), 221-239.

[15] P. A. Cruz, M. F. Tomé, I. W. Stewart, S. McKee, Numerical solution of the Ericksen-Leslie dynamic equations for two-dimensional nematic liquid crystal flows. J. Comput. Phys. 247 (2013), 109-136. 
[16] M. Doi, S. F. Edwards, The Theory of Polymer Dynamics, Oxford University Press, New York, 1986.

[17] A. M. Donald, A. H. Windle, S. Hanna. Liquid Crystalline Polymers, 2nd ed. Cambridge University Press, Cambridge, 2006.

[18] J. L. Ericksen, Anisotropic fluids, Arch. Rat. Mech. Anal., 4 (1960), 231-237 .

[19] J. L. Ericksen, Transversely isotropic fluids, Colloid Polym. Sci., 173(2) (1960), $117-122$.

[20] J. L. Ericksen, Theory of anisotropic fluids, Trans. Soc. Rheology, 4 (1960), 29-39.

[21] J. L. Ericksen, Conservation laws for liquid crystals, Trans. Soc. Rheol., 5 (1961), 23-34.

[22] V. G. Ferreira, A. C. Brandi, F. A. Kurokawa, P. Seleghim, A. Castelo, J. A. Cuminato, Incompressible turbulent flow simulation using k-e model and upwind schemes, Math. Probl. Eng., 2007 (2007), 1-26.

[23] M. G. Forest, R. Zhou, Q. Wang , Scaling behavior of kinetic orientational distributions for dilute nematic polymers in weak shear, J. Non-Newtonian Fluid. Mech., 116 (2004), 183-204.

[24] M. G. Forest, R. Zhou, Q. Wang, Chaotic boundaries of nematic polymers in mixed shear and extensional flows, Phys. Rev. Lett., 93 (2004), 088301.

[25] F. C. Frank, On The theory of liquid crystals, Discuss. Faraday Soc., 25 (1958), $19-28$.

[26] G. Friedel, Les états mésomorphes de la matiére, Ann. Phys. (Paris), 18 (1922), 273-474.

[27] P. G. de Gennes, Nematodynamics, in Molecular Fluids, R. Balian and G. Weill (Eds.), 373-400, Gordon and Breach, London, 1976.

[28] G. W. Gray, K. J. Harrison, J. A. Nash, New Family of Nematic Liquid Crystals for Displays, Electr. Lett., 9 (1973), 130-131.

[29] G. W. Gray, K. J. Harrison, J. A. Nash, J. Constant, D. S. Hulme, J. Kirton, E. P. Raynes, Stable, Low melting nematogens of positive dielectric anisotrpy for display devices, in Liquid Crystals and Ordered Fluids, J. F. Johnson, and R. S. Porter (Eds.), 2:617-643, Plenum Press, New York, 1974.

[30] F. H. Harlow, J. E. Welch, Numerical calculation of time-dependent viscous incompressible flow of fluid with free surface, Phys. Fluids, 8 (1965), 2182-2189.

[31] H. Heuer and H. Kneppe, F. Schneider, Flow of a nematic liquid crystal around a cylinder, Mol. Cryst. Liq. Cryst., 200 (1991), 51-70.

[32] S. Z. Hess, Fokker-Planck-equation approach to flow alignment in liquid crystals, Z. Naturforsch. A, 31 (1976), 1034-1037. 
[33] J. T. Jenkins, Flows of nematic liquid crystals, Ann. Rev. Fluid Mech., 10 (1978), 197-219.

[34] M. N. Kawaguchi, M. M. Denn, Visualization of the flow of a thermotropic liquid crystalline polymer in a tube with a conical contraction, J. Non-Newtonian Fluid. Mech., 69 (1997), 207-219.

[35] H. Kneppe, F. Schneider, N.K. Sharma, Rotational viscosity $\gamma_{1}$ of nematic liquid crystals, J. Chem. Phys., 77 (1982), 3203-3208.

[36] H. Kneppe, F. Schneider, Determination of the viscosity coefficients of the liquid crystal MBBA, Mol. Cryst. Liq. Cryst., 65 (1981), 23-38.

[37] H. Kneppe, F. Schneider and N.K. Sharma, A Comparative study of the viscosity coefficients of some nematic liquid crystals, Ber. Bunsenges. Phys. Chem., 85 (1981), 784-789.

[38] F. M. Leslie, Some constitutive equations for anisotropic fluids, Q. Jl. Mech. Appl. Math., 19 (1966), 357-370.

[39] F. M. Leslie, Some constitutive equations for liquid crystals, Arch. Rat. Mech. Anal., 28 (1968), 265-283.

[40] F. M. Leslie, Theory of flow phenomena in liquid crystals, Adv. Liq. Cryst., 4 (1979), 1-81.

[41] N. Mangiavacchi, A. Castelo, M. F. Tomé, J. A. Cuminato, M. L. B. Oliveira, S. McKee, An Effective Implementation of surface tension using the Marker and Cell method for axisymmetric and planar Flows, SIAM J. Sci. Comput., 26(4) (2005), 1340-1368.

[42] C. M. Oishi, M. F. Tomé, J. A. Cuminato, S. McKee, An implicit technique for solving 3D low Reynolds number moving free surface flows, J. Comput. Phys., 227 (2008), 7446-7468.

[43] C. M. Oishi, F. P. Martins, M. F. Tomé, J. A. Cuminato, S. McKee, Numerical solution of the eXtended Pom-Pom model for viscoelastic free surface flows, $J$. Non-Newtonian Fluid Mech., 166 (2011), 165-179.

[44] C. W. Oseen, Beitäge zur Theorie der anisotropen Flüssigkeiten, Arkiv För Matematik, Astronomi Och Fysik, 19A (1925), part 9, 1-19.

[45] C. W. Oseen, The Theory of liquid Crystals, Trans. Faraday Soc., 29 (1933), 883-899.

[46] O. Parodi, Stress tensor for a nematic liquid crystal, J. de Physique, 31 (1970), 581-584.

[47] P. Pieranski, E. Guyon, Transverse effects in nematic flows, Physics Letters, 49A (1974), 237-238.

[48] A. Rafiee, M. T. Manzari, M. Hosseini, An incompressible SPH method for simulation of unsteady viscoelastic free-surface flows, Int. J. Non-Linear Mech., 42 (2007), 1210-1223. 
[49] F. Reinitzer, Beitäge zur kenntnis des cholesterins, Monatsh. Chem., 9 (1888), 421-441.

[50] F. Reinitzer, Contributions to the knowledge of cholesterol. Translation of Reference [49], Liq. Cryst., 5 (1989), 7-18.

[51] A. D. Rey, M. M. Denn, Dynamical phenomena in liquid-crystalline materials, Annu. Rev. Fluid Mech., 34 (2002), 233-266.

[52] N. M. Ribe, A general theory of the dynamics of thin viscous sheets, J. Fluid Mech., 457 (2002), 255-283.

[53] N. M. Ribe, Coiling of viscous jets, Proceedings of the Royal Society of London Series A - Math. Phys. Eng. Sci., 460 (2004), 3223-3239.

[54] S. A. Roberts, R. R. Rao, Numerical simulations of mounding and submerging flows of shear-thinning jets impinging in a container, J. Non-Newtonian Fluid Mech., 166 (2011), 1100-1115.

[55] M. J. Stephen, J. P. Straley, Physics of liquid crystals, Rev. Mod. Phys., 46 (1974), 617-704.

[56] I. W. Stewart, The Static and Dynamic Continuum Theory of Liquid Crystals, Taylor and Francis, London, 2004.

[57] M. F. Tomé, S. McKee, GENSMAC: A computational marker-and-cell method for free surface flows in general domains, J. Comput. Phys., 110 (1994), 171-186.

[58] M.F. Tomé, B. Duffy, S. McKee, A numerical technique for solving unsteady non-Newtonian free surface flows, J. Non-Newtonian Fluid Mech., 62 (1996), 9-34.

[59] M. F. Tomé, A. Castelo, J. Murakami, J. A. Cuminato, R. Minghim, M.C.F. Oliveira, N. Mangiavacchi, S. McKee, Numerical simulation of axisymmetric free surface flows, J. Comput. Phys., 157 (2000), 441-472.

[60] M. F. Tomé, A. Castelo, J. A. Cuminato, N. Mangiavacchi, S. McKee, GENSMAC3D: A numerical method for solving unsteady three-dimensional free surface flows, Int. J. Num. Meth. Fluids, 37 (2001), 747-796.

[61] M. F. Tomé, N. Mangiavacchi, J. A. Cuminato, A. Castelo, S. McKee, A finite difference technique for simulating unsteady viscoelastic free surface flows, $J$. Non-Newtonian Fluid Mech., 106 (2002), 61-106.

[62] M. F. Tomé, L. Grossi, N. Mangiavacchi, A. Castelo, J. A. Cuminato, V. G. Ferreira, F. S. Souza, S. McKee, A numerical method for solving threedimensional generalized Newtonian free surface flows, J. Non-Newtonian Fluid Mech., 123 (2004), 85-103.

[63] M. F. Tomé, J. L. Doricio, J. A. Cuminato, A. Castelo, S. McKee, Solving viscoelastic free surface flow of a second order fluid using a marker-and-cell method approach, Int. J. Num. Meth. Fluids, 53 (2007), 599-627. 
[64] M. F. Tomé, A. Castelo, J. M. Nobrega, O. S. Carneiro, G. S. Paulo, F. T. Pereira, Numerical and experimental investigations of three-dimensional container filling with Newtonian viscous fluids, Comp. Fluids, 90 (2014), 172-185

[65] M. F. Tomé, J. Bertoco, C. M. Oishi, M. S. B. Araujo, D. Cruz, F. T. Pinho, M. Vynnycky, A finite difference technique for solving a time strain separable K-BKZ constitutive equation for two-dimensional moving free surface flows, $J$. Comput. Phys., 311 (2016), 114-141.

[66] B. Yang, A. Prosperetti, A second-order boundary-fitted projection method for free-surface flow computations, J. Comput. Phys., 231 (2006), 574-590. 